\title{
Chemical abundances of primary stars in the Sirius-like binary systems
}

\author{
X. M. Kong, ${ }^{1,2,3 \star}$ G. Zhao, ${ }^{1,3} \dagger$ J. K. Zhao, ${ }^{1}$ J. R. Shi, ${ }^{1,3}$ Y. Bharat Kumar, ${ }^{1}$ \\ L. Wang, ${ }^{1}$ J. B. Zhang, ${ }^{1}$ Y. Wang, ${ }^{1}$ and Y. T. Zhou ${ }^{1,3}$ \\ 1 Key Laboratory of Optical Astronomy, National Astronomical Observatories, Chinese Academy of Sciences, Beijing 100012, China \\ 2 School of Mechanical, Electrical and Information Engineering, Shandong University at Weihai, Weihai 264209, China \\ 3 School of Astronomy and Space Science, University of Chinese Academy of Sciences, Beijing 100049, China
}

Accepted XXX. Received YYY; in original form ZZZ

\begin{abstract}
Study of primary stars lying in Sirius-like systems with various masses of WD companions and orbital separations is one of the key aspects to understand the origin and nature of Barium (Ba) stars. In this paper, based on high resolution and high $\mathrm{S} / \mathrm{N}$ spectra, we present systematic analysis of photospheric abundances for $18 \mathrm{FGK}$ primary stars of Sirius-like systems including six giants and 12 dwarfs. Atmospheric parameters, stellar masses, and abundances of 24 elements (C, Na, Mg, Al, Si, S, K, $\mathrm{Ca}, \mathrm{Sc}, \mathrm{Ti}, \mathrm{V}, \mathrm{Cr}, \mathrm{Mn}, \mathrm{Fe}, \mathrm{Co}, \mathrm{Ni}, \mathrm{Cu}, \mathrm{Sr}, \mathrm{Y}, \mathrm{Zr}, \mathrm{Ba}, \mathrm{La}, \mathrm{Ce}$ and $\mathrm{Nd}$ ) are determined homogeneously. The abundance patterns in these sample stars show that most of the elements in our sample follow the behavior of field stars with similar metallicity. As expected, s-process elements in four known Ba giants show overabundance. A weak correlation was found between anomalies of s-process elemental abundance and orbital separation, suggesting the orbital separation of the binaries could not be the main constraint to differentiate strong Ba stars from mild Ba stars. Our study shows that the large mass $\left(>0.51 \mathrm{M}_{\odot}\right)$ of a WD companion in a binary system is not a sufficient condition to form a Ba star, even if the separation between the two components is small. Although not sufficient it seems to be a necessary condition since Ba stars with lower mass WDs in the observed sample were not found. Our results support that $[\mathrm{s} / \mathrm{Fe}]$ and [hs/ls] ratios of Ba stars are anti-correlated with the metallicity. However, the different levels of s-process overabundance among Ba stars may not to be dominated mainly by the metallicity.
\end{abstract}

Key words: stars: fundamental parameters - stars: abundances - stars: chemically peculiar- (stars:) binaries: general - (stars:) white dwarfs

\section{INTRODUCTION}

Sirius-like systems (SLSs), taking Sirius as the prototype, refer to white dwarfs (WDs) in binary or multiple star systems that contain at least one less luminous companion of spectral type K or earlier (Holberg et al. 2013). These systems play an important role in astrophysics, and are crucial for the studies of stellar and galactic evolution: 1) They are often used to investigate the initial-final mass relation (IFMR) among WDs (Zhao et al. 2012); 2) They are important to study the mass and mass-ratio properties of main-sequence stars in binary systems (Ferrario 2012); 3) Their study provides constraints on the physics of common-envelope evolu-

* Contact e-mail: xmkong@nao.cas.cn

† Contact e-mail: gzhao@nao.cas.cn tion (Zorotovic et al. 2010); 4) They are useful to study the progenitor model for type Ia supernovae (Wang et al. 2010); 5) They can be used to investigate the origin and nature of Ba stars.

Barium II (Ba) stars are G-K type giants (e.g. Bidelman \& Keenan 1951) and dwarfs (e.g. Tomkin et al. 1989) that are spectroscopically characterized by overabundance of Ba and other s-process elements. The s-process nucleosynthesis is expected in the interiors of thermally pulsating Asymptotic Giant Branch (TP-AGB) stars followed by deep dredge-up phenomena, named third dredge-up, which brings the processed materials to the surface. However, the luminosity and temperature estimates for the Ba stars are not expected for them to evolve far enough to be able to produce the s-process elements in their interiors, thus they cannot be self-enriched (Smiljanic et al. 2007). In the 
early eighties, McClure et al. (1980) discovered that all Ba giants are likely members of binary systems through radial velocity monitoring. Further studies (McClure 1983; Boffin \& Jorissen 1988; Jorissen et al. 1998) showed that the companions could be white dwarfs. So, it is generally believed that Ba stars belong to binary systems and the chemical peculiarities observed in them are due to mass transfer from their AGB companions (now WD companions) during TP-AGB phase. Therefore, a binary system with a WD companion appears to be a necessary condition for producing a Ba star. Consequently, a series of critical questions aroused the scholars' enormous interest. One is that whether the existence of a WD companion in a binary system is sufficient to produce a Ba star. The identification of some normal giants with WD companions gave a negative answer to this question (Jorissen \& Boffin 1992; Zacs et al. 1997; Merle et al. 2014), suggested to look for other important factors to understand the chemical peculiarities observed in Ba stars. Boffin \& Jorissen (1988) suggested that the degree of chemical contamination in Ba stars depends on the mass of the WD companion and the orbital separation in the binaries. Hurley et al. (2000) predicted that the minimum WD mass should be at least $0.51 \mathrm{M}_{\odot}$ so as to its progenitor can evolve to the AGB phase. Merle et al. (2016, hereafter MER16) studied 11 binary systems involving WD companions of various masses, wherein three primary stars with WD companions of masses lower than about $0.5 \mathrm{M}_{\odot}$ indeed did not show s-process overabundances, and six giants among eight sample stars with WD companions of mass above $0.5 \mathrm{M}_{\odot}$ showed clear s-process enrichment. Such study supports the prediction of Hurley et al. (2000), however, the constraints are provided based on few observed data points and hence required larger sample to draw any firm conclusion.

The level of chemical enrichment in Ba stars and connection with their orbital period is investigated extensively and showed that the degree of Ba pollution is strongly correlated with the orbital period and mild $\mathrm{Ba}$ stars should have wider orbital separations than classical Ba stars (Zacs 1994; Han et al. 1995; Antipova et al. 2004; Yang et al. 2016). On the other hand, Jorissen et al. (1998) suggested that the difference between mild and strong Ba stars may not be dominated by the orbital separation, and metallicity could play important role. This argument supports the study of Kovacs (1985) who suggested that increase in Ba enrichment in stars follows with iron deficiency. Smiljanic et al. (2007) claimed that the neutron exposure during the s-process operation may play an important role. Other parameters, such as eccentricity, mass-loss mechanism (wind accretion or Roche lobe overflow), the efficiency of thermal pulses and dilution factors, also play role in observed chemical peculiarities of Ba stars (Van der Swaelmen et al. 2017; Busso et al. 2001; Allen \& Barbuy 2006; de Castro et al. 2016).

In general, the origin of s-process elements enrichment in Ba stars is still not well understood and lacks a definite conclusion. Detailed abundance analysis of large sample of binaries with a variety of orbital periods and masses of WD companions may give clues to their origin. In this paper, we analyzed the elemental abundances of 18 FGK primary stars in SLSs including four known Ba giants, two giants and 12 dwarfs. Together with three Ba dwarfs lying in SLSs which have been reported in Kong et al. (2018) (hereafter paper I), we compared the abundance patterns from this study with the general trends observed among normal disk stars from literature, and discussed the role of WD companion masses, orbital period and the metallicity in the levels of s-process overabundance in Ba stars. Our paper is organized as follows, Section 2 presents the sample selection. Observations and process of data reduction are described in Section 3. Analysis and results are presented in Section 4. The results are discussed in Section 5, and conclusions are drawn in Section 6.

\section{SAMPLE SELECTION}

We have selected 21 primary stars ( including BD $+68^{\circ} 1027$, RE J0702+129 and BD+80 670 from Paper I) of FGK type in SLSs that are located in northern hemisphere, with apparent magnitudes brighter than 11, from the 98 candidates in the solar neighborhood provided in a catalogue by Holberg et al. (2013). The sample are covered from spectroscopic binaries to wide binaries with various masses of WD companions. Table 1 provides the basic information of 21 sample comes from Holberg et al. (2013). The successive columns present the primary star name, spectral type of the primary, V magnitude from Tycho satellite, parallax from Hipparcos, Distance in pc (calculated from Hipparcos parallax or from Holberg et al. (2013)), parallax from Gaia, Distance in pc (calculated from Gaia parallax), the mass of the WD in solar mass, binary separation in arcseconds, the estimated semimajor axis ( $a$, in au), measured or estimated orbital periods ( $p$, in yr), orbital eccentricity, the number of known components in each system, and the name of spectrograph used to take spectra.

\section{OBSERVATIONS AND DATA REDUCTION}

The observations of entire sample were carried out using two different telescopes: i) spectra of 16 stars were obtained with the ARC Echelle Spectrograph (ARCES) attached to the $3.5 \mathrm{~m}$ telescope at the Apache Point Observatory (APO), during three runs of October, November 2014 and February 2015. The ARCES spectral resolving power is $R \sim 31500$ with wavelength coverage from $4400 \AA$ to $10000 \AA$; ii) spectra of six stars were taken using the Coudé Echelle Spectrograph (CES) attached to the $2.16 \mathrm{~m}$ telescope operated by National Astronomical Observatories (Xinglong, China), during March and September 2015. The spectra have resolution $R \sim 50000$ and cover the wavelength range $4000 \AA$ to $9000 \AA$. For all samples, the exposure time was chosen in order to obtain a $\mathrm{S} / \mathrm{N}$ of at least 100 over the entire spectral range. In addition, the solar spectra obtained from the two telescopes were used to perform differential abundance analysis. The raw spectra were processed in a standard procedure following Paper I. Figure 1 present the spectra of a dwarf star and a giant star showing main features of absorption in the wavelength region from $6140 \AA$ to $6175 \AA$.

To check the consistency, the spectra of BD-01469 taken from two observational set-ups are used to measure EWs of common lines, and are compared (See Figure 2). Note, the spectra observed in two set-ups showing good consistency. The systematic difference between the data of two sets is small and a linear regression was obtained, $E W_{3.5 \mathrm{~m}}=$ 
Table 1. Basic information of 21 program stars. Top: sample stars which have abundance analysis in previous literatures; middle: three Ba dwarfs analysed by Paper I; bottom: sample stars analysed for the first time.

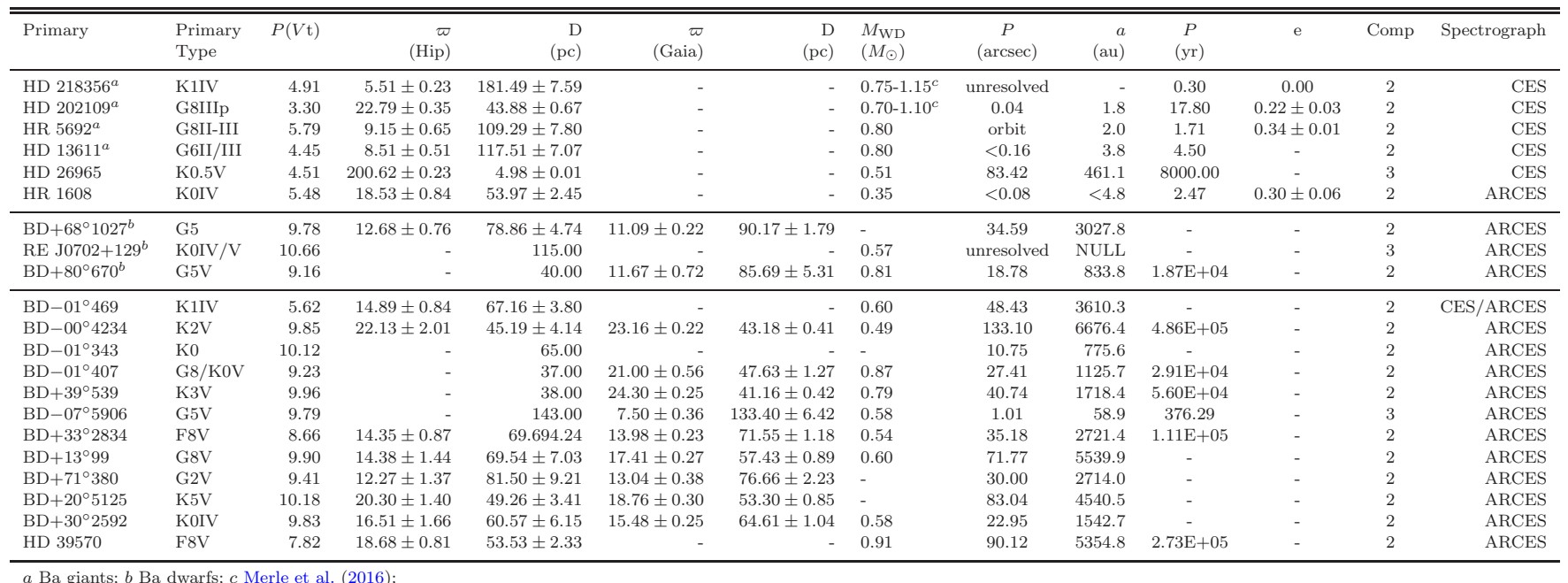

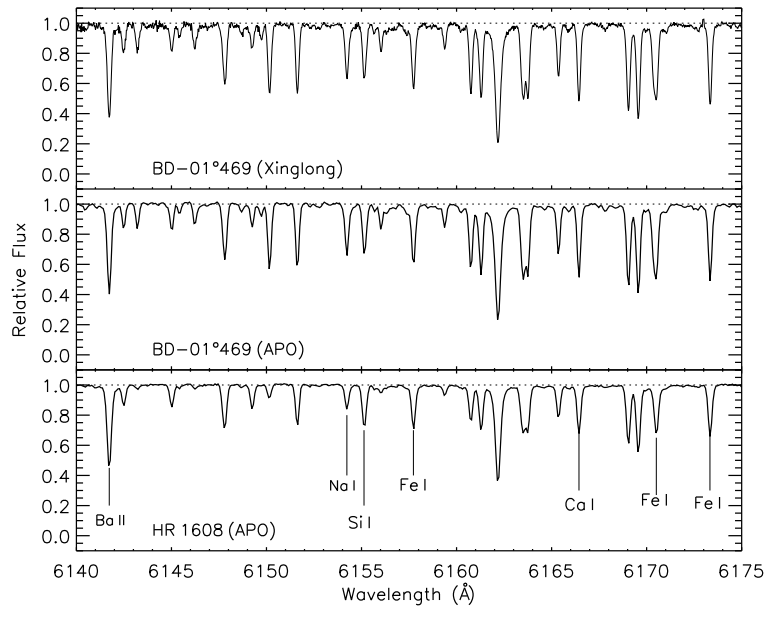

Figure 1. Sample spectra of the normalization of the continuum in the wavelength region from 6140 to $6175 \AA$.

$1.002( \pm 0.006) E W_{2.16 \mathrm{~m}}+0.162( \pm 0.523)(\mathrm{m \AA})$. The standard deviation is about $2.8 \mathrm{m \AA}$.

\section{ANALYSIS AND RESULTS}

\subsection{Determination of atmospheric parameters}

Atmospheric parameters like effective temperature $\left(T_{\text {eff }}\right)$, surface gravity $(\log g)$, metallicity $([\mathrm{Fe} / \mathrm{H}])$ and microturbulence $\left(\xi_{t}\right)$, are determined in a similar manner that was described in Paper I. The $T_{\text {eff }}$ was obtained by imposing excitation equilibrium, i.e, all Fe I lines with different excitation potentials provide the same abundance. The microturbulent velocity $\left(\xi_{t}\right)$ was determined by forcing that iron abundances from Fe I lines of different strengths show no dependence on their equivalent widths (EWs). A linear fit was searched where the angular coefficient is close to zero, and the uncertainty in this coefficient indicates the uncertainty of the $T_{\text {eff }}$ (spec) and $\xi_{t}$. To estimate $1 \sigma$ uncertainty of these

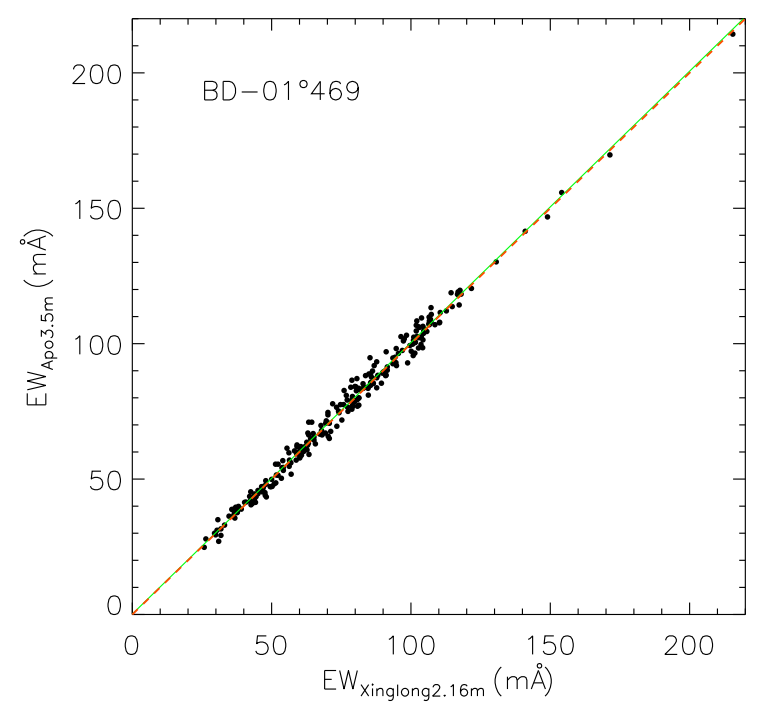

Figure 2. A comparison of equivalent widths $(\mathrm{m} \AA$ ) obtained by Xinglong $(2.16 \mathrm{~m})$ with $\mathrm{APO}(3.5 \mathrm{~m})$ for star $\mathrm{BD}-01^{\circ} 469$ in common. The solid line is a linear fit to the points, whereas the dotted line is the one-to-one relation.

parameters we change their values, respectively, in the plots of Fe I abundance against excitation potential and EWs, until the angular coefficient of the linear fit match their own uncertainty. The errors of $T_{\text {eff }}$ (spec) and $\xi_{t}$ estimated by this method are listed in Table 2, and the typical errors are about $\pm 50 \mathrm{~K}$ and $0.15 \mathrm{Kms}^{-1}$, respectively.

For comparison, photometric temperatures are derived from $V-K$ colour and empirical calibration relations given by Alonso et al. $(1996,1999,2001)$. Two error sources have been taken into account. One is from the errors of Ks, collected from the Two Micron All-Sky Survey (Cutri et al. 2003), and the other is the measured errors estimated by Alonso et al. (1996, 1999)(see in Table 2).

For the stars with available parallaxes, the effective 
temperatures derived from two methods are compared and shown in Figure 3. For BD $-01^{\circ} 469$ and $\mathrm{BD}-07^{\circ} 5906$, the difference in temperatures between two methods are as high as $366 \mathrm{~K}$ and $260 \mathrm{~K}$. For $\mathrm{BD}-01^{\circ} 469$, such large error in temperature is probably propagated from error in $K_{\mathrm{s}}$ band magnitude of $0.234 \mathrm{mag}$, which is estimated to be $170 \mathrm{~K}$. For $\mathrm{BD}-07^{\circ} 5906$, the cause for the big difference could be its second companion, which may influence its V or K magnitude. The mean difference between the two methods for other sample stars are $\left\langle T_{\text {eff }}(\right.$ spec $\left.)-T_{\text {eff }}(V-K)\right\rangle$, is $22 \pm 79 \mathrm{~K}$. Table 2 list the effective temperatures derived from two methods, and we have adopted the $T_{\text {eff }}(s p e c)$ for abundance analysis.

For stars with available parallaxes, we also derived $\log g$ using standard relation involves mass, temperature, and bolometric flux:

$\log g=\log g_{\odot}+\log \left(\frac{M}{M_{\odot}}\right)+4 \log \left(\frac{T_{\text {eff }}}{T_{\text {eff } \odot}}\right)+0.4\left(M_{\mathrm{bol}}-M_{\mathrm{bol} \odot}\right)$

$M_{\mathrm{bol}}=V_{\mathrm{mag}}+B C+5 \log \varpi+5-A_{\mathrm{V}}$

where $\mathrm{M}$ is the stellar mass, which is estimated using an interpolator of the evolutionary tracks of Yi et al. (2003). The bolometric corrections were calculated using the relation given by Alonso et al. $(1995,1999)$. For stars with both Gaia and Hipparcos parallaxes, we adopted Gaia parallaxes due to their higher accuracy. From Table 1, we can see that the accuracy of parallaxes in our program stars are high with errors less than 7\%. According to equations (1) and (2), this corresponds to an error of 0.06 dex in $\log g$. Further contributions to the error come from the uncertainty in $V_{\mathrm{mag}}$, effective temperature and parallax, for which we add 0.04 dex. Finally, the total error estimated for surface gravity is about 0.1 dex.

For comparison, we also calculated the $\log g$ using ionization equilibrium method wherein the value of $\log g$ is obtained by forcing the FeI and Fe II lines to yield the same iron abundance (See Figure 3). Table 2 list the surface gravities derived from both methods. The difference of surface gravities between the two methods is small with a mean value of $0.03 \pm 0.10$ dex. For star $\mathrm{BD}-01^{\circ} 343$, which has no parallaxes from Hipparcos or Gaia, we adopted $\log g$ (Spec) for further analysis. For the rest of our sample, we adopted $\log g$ (Parallax).

The initial metallicity values for most of our program stars were set to $[\mathrm{Fe} / \mathrm{H}]=0.0$, and this value will not affect the final result. For the stars with available metallicities in literature, we have taken them as initial metallicities. The final results was adopted by iterating the whole process of determining the atmospheric parameters $T_{\text {eff }}, \log g,[\mathrm{Fe} / \mathrm{H}]$, and $\xi_{t}$ until they were consistent, and are given in Table 2. The error estimated in $[\mathrm{Fe} / \mathrm{H}]$ is \pm 0.1 dex, which was calculated as described in Ryan et al. (1996).

\subsection{Atomic data, Abundances, and Error analysis}

Atomic data for various elements used in this study are the same as those given in paper I, and we added Sulphur (S I) lines from Meléndez et al. (2014). We have performed the abundance analysis using EWs, and a grid of
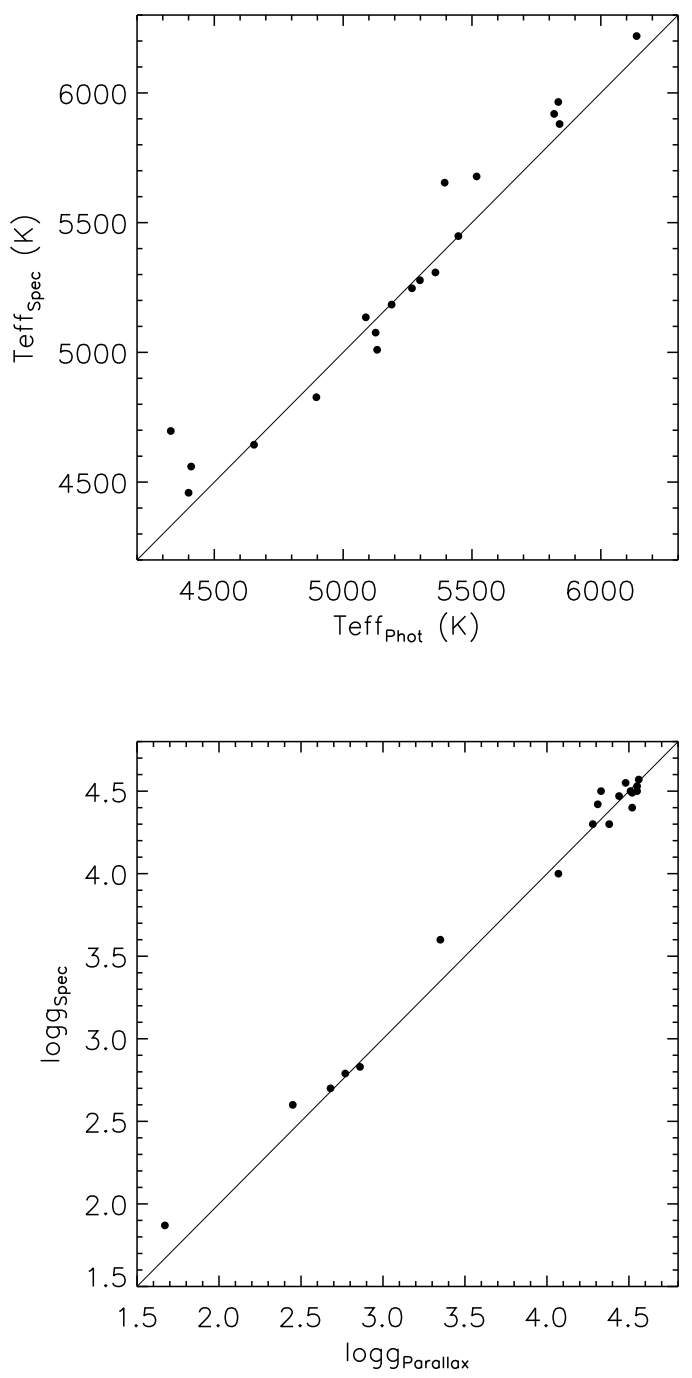

Figure 3. In the top panel, we show the comparison of effective temperatures derived from the photometric colour index $V-K$ and excitation equilibrium methods. In the bottom panel, the comparison of surface gravities obtained by Gaia or Hipparcos parallaxes (X axis) and ionization balance of Fe I and Fe II lines ( $\mathrm{Y}$ axis) are presented.

plane-parallel and local thermodynamic equilibrium models provided by Kurucz (1993). The ABONTEST8 program supplied by Dr. P. Magain was used to calculate the theoretical line EWs, and elemental abundances were calculated by requiring that the calculated equivalent width from the model should match the observed value (see paper I for details). Differential abundances $([\mathrm{X} / \mathrm{Fe}])$ are obtained relative to solar values, wherein we adopted $T_{\text {eff }}=5780 \mathrm{~K}, \log g=$ 4.44 , and $\xi_{t}=0.9 \mathrm{~km} \mathrm{~s}^{-1}$ to derive solar abundances. The atomic line data and corresponding EWs, and abundances for our program stars are listed in Table 3 . The complete table is available in electronic form.

The uncertainties in abundance measurements are estimated by considering the uncertainties in stellar parameters 
Table 2. The basic stellar parameters for 21 sample stars.

\begin{tabular}{|c|c|c|c|c|c|c|c|c|c|c|}
\hline Primary & $\mathrm{V}-\mathrm{K}$ & $\mathrm{E}(\mathrm{V}-\mathrm{K})$ & $M_{\mathrm{v}}$ & $M_{\mathrm{p}} / M_{\odot}$ & $\begin{array}{l}T_{\text {eff }} \\
(\mathrm{V}-\mathrm{K})\end{array}$ & $\begin{array}{c}T_{\text {eff }} \\
\text { (Spec) }\end{array}$ & $\begin{array}{c}\log g \\
\text { (Parallax) }\end{array}$ & $\begin{array}{c}\log g \\
(\mathrm{Spec})\end{array}$ & {$[\mathrm{Fe} / \mathrm{H}]$} & $\xi_{\mathrm{t}}$ \\
\hline HD 218356 & 2.99 & 0.25 & -1.53 & 2.50 & $4400 \pm 76$ & $4459 \pm 74$ & 1.67 & 1.87 & -0.43 & $1.9 \pm 0.09$ \\
\hline HD 202109 & 2.03 & 0.04 & -0.02 & 3.20 & $5132 \pm 117$ & $5010 \pm 50$ & 2.68 & 2.70 & -0.01 & $1.7 \pm 0.05$ \\
\hline HR 5692 & 2.07 & 0.08 & 0.50 & 2.80 & $5126 \pm 361$ & $5076 \pm 46$ & 2.86 & 2.83 & 0.02 & $1.3 \pm 0.06$ \\
\hline HR 1608 & 1.79 & 0.07 & 1.73 & 1.90 & $5447 \pm 336$ & $5448 \pm 45$ & 3.35 & 3.60 & -0.09 & $1.3 \pm 0.05$ \\
\hline HD 13611 & 2.04 & 0.10 & -0.99 & 3.80 & $5188 \pm 119$ & $5184 \pm 50$ & 2.45 & 2.60 & -0.01 & $1.5 \pm 0.05$ \\
\hline HD 26965 & 2.00 & 0.01 & 5.93 & 0.85 & $5088 \pm 112$ & $5135 \pm 45$ & 4.51 & 4.50 & -0.38 & $0.7 \pm 0.06$ \\
\hline $\mathrm{BD}+68^{\circ} 1027$ & 1.49 & 0.04 & 5.01 & 0.93 & $5819 \pm 32$ & $5919 \pm 55$ & 4.48 & 4.55 & -0.31 & $1.0 \pm 0.10$ \\
\hline RE J0702+129 & 2.06 & 0.03 & 5.28 & 0.93 & $5052 \pm 42$ & $5531 \pm 60$ & 4.43 & 4.20 & -0.06 & $1.9 \pm 0.12$ \\
\hline $\mathrm{BD}+80^{\circ} 670$ & 1.57 & 0.13 & 4.50 & 1.05 & $5840 \pm 41$ & $5880 \pm 60$ & 4.33 & 4.50 & 0.13 & $1.6 \pm 0.10$ \\
\hline $\mathrm{BD}-01^{\circ} 469$ & 2.93 & 0.09 & 1.48 & 1.40 & $4331 \pm 170$ & $4697 \pm 55$ & 2.77 & 2.79 & -0.09 & $1.2 \pm 0.06$ \\
\hline $\mathrm{BD}-00^{\circ} 4234$ & 2.66 & 0.02 & 6.57 & 0.56 & $4410 \pm 44$ & $4560 \pm 56$ & 4.38 & 4.30 & -0.96 & $0.3 \pm 0.40$ \\
\hline $\mathrm{BD}-01^{\circ} 343$ & 1.86 & 0.02 & - & - & $5292 \pm 46$ & $5260 \pm 70$ & - & 4.30 & 0.36 & $0.9 \pm 0.15$ \\
\hline $\mathrm{BD}-1^{\circ} 407$ & 1.88 & 0.06 & 5.54 & 0.83 & $5298 \pm 47$ & $5278 \pm 55$ & 4.52 & 4.40 & -0.12 & $0.8 \pm 0.12$ \\
\hline $\mathrm{BD}+39^{\circ} 0539$ & 2.48 & 0.01 & 6.77 & 0.78 & $4654 \pm 40$ & $4644 \pm 54$ & 4.56 & 4.57 & 0.01 & $0.4 \pm 0.25$ \\
\hline $\mathrm{BD}-7^{\circ} 5906$ & 1.80 & 0.07 & 4.09 & 0.92 & $5394 \pm 48$ & $5654 \pm 44$ & 4.07 & 4.00 & -0.13 & $0.9 \pm 0.10$ \\
\hline $\mathrm{BD}+33^{\circ} 2834$ & 1.28 & 0.02 & 4.37 & 1.17 & $6139 \pm 40$ & $6219 \pm 65$ & 4.44 & 4.47 & -0.06 & $1.2 \pm 0.08$ \\
\hline $\mathrm{BD}+13^{\circ} 99$ & 1.92 & 0.08 & 6.00 & 0.73 & $5267 \pm 51$ & $5247 \pm 50$ & 4.55 & 4.53 & -0.38 & $0.6 \pm 0.15$ \\
\hline $\mathrm{BD}+71^{\circ} 380$ & 1.68 & 0.05 & 4.92 & 0.73 & $5518 \pm 45$ & $5678 \pm 45$ & 4.31 & 4.42 & -0.41 & $0.7 \pm 0.13$ \\
\hline $\mathrm{BD}+20^{\circ} 5125$ & 2.21 & 0.02 & 6.44 & 0.79 & $4896 \pm 41$ & $4827 \pm 60$ & 4.55 & 4.50 & -0.14 & $0.3 \pm 0.30$ \\
\hline $\mathrm{BD}+30^{\circ} 2592$ & 1.79 & 0.02 & 5.67 & 0.89 & $5358 \pm 43$ & $5308 \pm 50$ & 4.52 & 4.49 & -0.07 & $0.9 \pm 0.13$ \\
\hline HD 39570 & 1.48 & 0.03 & 4.11 & 1.25 & $5835 \pm 39$ & $5965 \pm 35$ & 4.28 & 4.30 & 0.03 & $1.3 \pm 0.05$ \\
\hline
\end{tabular}

Table 3. Atomic line data and measured EWs and absolute abundances for our program stars.

\begin{tabular}{clccccrc}
\hline \hline Star & Ele & Ion & $\begin{array}{c}\text { Wavelength } \\
{[\AA]}\end{array}$ & $\log g f$ & $\begin{array}{r}E_{\text {low }} \\
{[\mathrm{eV}]}\end{array}$ & $\begin{array}{r}\text { EW } \\
(\mathrm{m} \AA)\end{array}$ & $\begin{array}{r}\text { Abun } \\
(\mathrm{dex})\end{array}$ \\
\hline $\mathrm{BD}-01^{\circ} 469$ & $\mathrm{C}$ & 1 & 5380.337 & 7.680 & -1.570 & 13.4 & 8.398 \\
& $\mathrm{Na}$ & 1 & 6160.750 & 2.100 & -1.240 & 101.0 & 6.326 \\
& $\mathrm{Na}$ & 1 & 6154.230 & 2.100 & -1.510 & 86.5 & 6.375 \\
& $\mathrm{Mg}$ & 1 & 7691.550 & 5.750 & -1.000 & 112.1 & 7.699 \\
& $\mathrm{Mg}$ & 1 & 7387.690 & 5.750 & -1.250 & 97.0 & 7.773 \\
& $\mathrm{Mg}$ & 1 & 8717.830 & 5.930 & -1.050 & 98.2 & 7.735 \\
& $\mathrm{Mg}$ & 1 & 8712.690 & 5.930 & -1.310 & 77.5 & 7.769 \\
\hline
\end{tabular}

and the measurement errors of EWs. Table 4 and Table 5 list the abundance differences when changing the effective temperature by $50 \mathrm{~K}$, the surface gravity by $0.1 \mathrm{dex}$, the iron abundance by $0.1 \mathrm{dex}$, and the microturbulent velocity by $0.15 \mathrm{~km} \mathrm{~s}^{-1}$. $\sigma_{\text {stat }}$ represents the statistical uncertainty which is line-to-line dispersion divided by $\sqrt{N}$, where $\mathrm{N}$ is the number of lines used for a given element. The total error was calculated by taking square root of quadratic sum of the errors associated to all factors, and are given in Col. 8 of Table $4 \& 5$. These computations for two stars with different characteristics, giant (close binaries) and dwarf (wide binaries), are given in Table 4 and Table 5, respectively.

We note that the uncertainties in Table $4 \& 5$ are the uncertainties for absolute abundance $([\mathrm{X} / \mathrm{H}])$. To estimate the uncertainties on the $[\mathrm{X} / \mathrm{Fe}]$ abundance, the absolute abundances $\mathrm{A}(\mathrm{Fe})$ and $\mathrm{A}(\mathrm{X})$ were quadratically added and listed in Table 8 , and also showed in respective figures.

\subsection{Comparison with previous studies}

Elemental abundances for six stars in our sample have been determined previously in literature, in which four stars
Table 4. Abundance errors of giant HR 1608.

\begin{tabular}{lrrrrrrr}
\hline \hline$\Delta[\mathrm{X} / \mathrm{H}]$ & $N$ & $\sigma_{\text {stat }}$ & $\begin{array}{r}\Delta T_{\text {eff }} \\
(+50 \mathrm{~K})\end{array}$ & $\begin{array}{r}\Delta \log g \\
(+0.1)\end{array}$ & $\begin{array}{r}\Delta[\mathrm{Fe} / \mathrm{H}] \\
(+0.1)\end{array}$ & $\begin{array}{r}\Delta \xi_{\mathrm{t}} \\
\left(+0.15 \mathrm{~km} \mathrm{~s}^{-1}\right)\end{array}$ & $\sigma_{\text {Total }}$ \\
\hline$\Delta[\mathrm{C} / \mathrm{H}]$ & 1 & - & -0.04 & 0.03 & -0.01 & 0.00 & 0.05 \\
$\Delta[\mathrm{Na} / \mathrm{H}]$ & 3 & 0.06 & 0.03 & -0.01 & 0.00 & -0.02 & 0.07 \\
$\Delta[\mathrm{Mg} / \mathrm{H}]$ & 5 & 0.02 & 0.02 & -0.02 & 0.01 & -0.02 & 0.04 \\
$\Delta[\mathrm{Al} / \mathrm{H}]$ & 6 & 0.03 & 0.02 & -0.02 & 0.00 & -0.01 & 0.04 \\
$\Delta[\mathrm{Si} / \mathrm{H}]$ & 23 & 0.02 & 0.01 & 0.00 & 0.01 & -0.02 & 0.03 \\
$\Delta[\mathrm{S} / \mathrm{H}]$ & 3 & 0.03 & -0.02 & 0.03 & 0.00 & -0.01 & 0.05 \\
$\Delta[\mathrm{K} / \mathrm{H}]$ & 1 & - & 0.05 & -0.04 & 0.03 & -0.05 & 0.09 \\
$\Delta[\mathrm{Ca} / \mathrm{H}]$ & 16 & 0.02 & 0.04 & -0.02 & 0.00 & -0.05 & 0.07 \\
$\Delta[\mathrm{ScI} / \mathrm{H}]$ & 3 & 0.01 & 0.06 & 0.00 & 0.01 & -0.01 & 0.06 \\
$\Delta[\mathrm{ScII} / \mathrm{H}]$ & 5 & 0.01 & 0.00 & 0.04 & 0.03 & -0.04 & 0.06 \\
$\Delta[\mathrm{TiI} / \mathrm{H}]$ & 22 & 0.02 & 0.06 & -0.01 & 0.01 & -0.05 & 0.08 \\
$\Delta[\mathrm{TiII} / \mathrm{H}]$ & 7 & 0.03 & 0.00 & 0.04 & 0.03 & -0.05 & 0.07 \\
$\Delta[\mathrm{V} / \mathrm{H}]$ & 7 & 0.03 & 0.06 & 0.00 & 0.01 & -0.02 & 0.07 \\
$\Delta[\mathrm{CrI} / \mathrm{H}]$ & 9 & 0.01 & 0.05 & -0.01 & 0.01 & -0.03 & 0.06 \\
$\Delta[\mathrm{CrII} / \mathrm{H}]$ & 5 & 0.03 & -0.02 & 0.04 & 0.02 & -0.04 & 0.07 \\
$\Delta[\mathrm{Mn} / \mathrm{H}]$ & 3 & 0.03 & 0.05 & -0.02 & 0.01 & -0.07 & 0.09 \\
$\Delta[\mathrm{FeI} / \mathrm{H}]$ & 172 & 0.01 & 0.04 & -0.01 & 0.00 & -0.05 & 0.06 \\
$\Delta[\mathrm{FeII} / \mathrm{H}]$ & 19 & 0.02 & -0.02 & 0.04 & 0.03 & -0.05 & 0.07 \\
$\Delta[\mathrm{Co} / \mathrm{H}]$ & 8 & 0.04 & 0.05 & 0.00 & 0.01 & -0.02 & 0.07 \\
$\Delta[\mathrm{Ni} / \mathrm{H}]$ & 42 & 0.01 & 0.04 & 0.00 & 0.01 & -0.04 & 0.05 \\
$\Delta[\mathrm{Cu} / \mathrm{H}]$ & 4 & 0.05 & 0.04 & -0.01 & 0.01 & -0.07 & 0.10 \\
$\Delta[\mathrm{Sr} / \mathrm{H}]$ & 1 & - & 0.06 & -0.01 & 0.01 & -0.08 & 0.10 \\
$\Delta[\mathrm{Y} / \mathrm{H}]$ & 2 & 0.03 & 0.00 & 0.04 & 0.03 & -0.07 & 0.09 \\
$\Delta[\mathrm{Zr} / \mathrm{H}]$ & 1 & - & 0.00 & 0.04 & 0.03 & -0.01 & 0.05 \\
$\Delta[\mathrm{Ba} / \mathrm{H}]$ & 3 & 0.06 & 0.02 & 0.01 & 0.05 & -0.08 & 0.11 \\
$\Delta[\mathrm{La} / \mathrm{H}]$ & 3 & 0.04 & 0.01 & 0.04 & 0.04 & -0.01 & 0.07 \\
$\Delta[\mathrm{Ce} / \mathrm{H}]$ & 2 & 0 & 0.01 & 0.04 & 0.03 & -0.05 & 0.07 \\
$\Delta[\mathrm{Nd} / \mathrm{H}]$ & 3 & 0.02 & 0.01 & 0.04 & 0.04 & -0.02 & 0.06 \\
\hline & & & & & & &
\end{tabular}

(HD 218356, HD 202109, HR 5692 and HR 1608) have been studied by MER16. Atmospheric parameters and abundances of these stars are listed in Table $6 \& 7$. From Table 6 it is clear that derived stellar parameters for five sample stars in this study are in good agreement with literature. For HD 218356, there lies obvious difference in $T_{\text {eff }}, \log g$ and $\xi_{t}$ compare to MER16. However, the methodology employed by MER16 to derive atmosphere parameters are similar to ours; excitation potentials equilibrium method for $T_{\text {eff }}$ and ionization equilibrium method for $\log g$. MER16's estimates of temperature, surface gravity and microturbulence are 4244 
Table 5. Abundance errors of dwarf $\mathrm{BD}+71^{\circ} 380$.

\begin{tabular}{lrrrrrrr}
\hline \hline$\Delta[\mathrm{X} / \mathrm{H}]$ & $\mathrm{N}$ & $\sigma_{\text {stat }}$ & $\begin{array}{r}\Delta T_{\text {eff }} \\
(+50 \mathrm{~K})\end{array}$ & $\begin{array}{r}\Delta \log g \\
(+0.1)\end{array}$ & $\begin{array}{r}\Delta[\mathrm{Fe} / \mathrm{H}] \\
(+0.1)\end{array}$ & $\begin{array}{r}\Delta \xi_{\mathrm{t}} \\
\left(+0.15 \mathrm{~km} \mathrm{~s}^{-1}\right)\end{array}$ & $\sigma_{\text {Total }}$ \\
\hline$\Delta[\mathrm{C} / \mathrm{H}]$ & 1 & - & 0.03 & 0.03 & -0.01 & 0.00 & 0.04 \\
$\Delta[\mathrm{Na} / \mathrm{H}]$ & 3 & 0.02 & -0.03 & -0.02 & 0.00 & -0.01 & 0.04 \\
$\Delta[\mathrm{Mg} / \mathrm{H}]$ & 6 & 0.01 & -0.03 & -0.02 & 0.01 & -0.01 & 0.04 \\
$\Delta[\mathrm{Al} / \mathrm{H}]$ & 4 & 0.03 & -0.02 & -0.01 & 0.00 & 0.00 & 0.04 \\
$\Delta[\mathrm{Si} / \mathrm{H}]$ & 21 & 0.01 & -0.01 & -0.01 & 0.01 & -0.01 & 0.02 \\
$\Delta[\mathrm{K} / \mathrm{H}]$ & 1 & - & -0.05 & -0.05 & 0.02 & -0.02 & 0.07 \\
$\Delta[\mathrm{Ca} / \mathrm{H}]$ & 11 & 0.02 & -0.04 & -0.03 & 0.01 & -0.02 & 0.06 \\
$\Delta[\mathrm{Sc} / \mathrm{H}]$ & 4 & 0.03 & -0.01 & 0.03 & 0.03 & -0.02 & 0.06 \\
$\Delta[\mathrm{TiI} / \mathrm{H}]$ & 22 & 0.02 & -0.06 & -0.01 & 0.00 & -0.03 & 0.07 \\
$\Delta[\mathrm{TiII} / \mathrm{H}]$ & 5 & 0.03 & -0.01 & 0.03 & 0.03 & -0.02 & 0.06 \\
$\Delta[\mathrm{V} / \mathrm{H}]$ & 5 & 0.04 & -0.06 & 0.00 & 0.00 & -0.01 & 0.07 \\
$\Delta[\mathrm{CrI} / \mathrm{H}]$ & 8 & 0.02 & -0.05 & -0.02 & 0.01 & -0.02 & 0.05 \\
$\Delta[\mathrm{CrII} / \mathrm{H}]$ & 6 & 0.03 & 0.01 & 0.03 & 0.02 & -0.02 & 0.05 \\
$\Delta[\mathrm{Mn} / \mathrm{H}]$ & 4 & 0.03 & -0.04 & -0.02 & 0.00 & -0.02 & 0.06 \\
$\Delta[\mathrm{FeI} / \mathrm{H}]$ & 124 & 0.01 & 0.04 & -0.01 & 0.00 & -0.03 & 0.05 \\
$\Delta[\mathrm{FeII} / \mathrm{H}]$ & 16 & 0.02 & -0.01 & 0.03 & 0.03 & -0.03 & 0.05 \\
$\Delta[\mathrm{Co} / \mathrm{H}]$ & 5 & 0.03 & -0.04 & 0.00 & 0.00 & -0.01 & 0.05 \\
$\Delta[\mathrm{Ni} / \mathrm{H}]$ & 34 & 0.01 & -0.04 & -0.01 & 0.01 & -0.02 & 0.04 \\
$\Delta[\mathrm{Cu} / \mathrm{H}]$ & 2 & 0.02 & -0.04 & -0.02 & 0.01 & -0.03 & 0.06 \\
$\Delta[\mathrm{Sr} / \mathrm{H}]$ & 1 & - & -0.05 & -0.01 & 0.00 & -0.03 & 0.06 \\
$\Delta[\mathrm{Y} / \mathrm{H}]$ & 3 & 0.03 & -0.01 & 0.04 & 0.03 & -0.03 & 0.06 \\
$\Delta[\mathrm{Ba} / \mathrm{H}]$ & 3 & 0.04 & -0.02 & 0.01 & 0.04 & -0.05 & 0.08 \\
$\Delta[\mathrm{La} / \mathrm{H}]$ & 1 & - & -0.02 & 0.04 & 0.03 & -0.01 & 0.05 \\
$\Delta[\mathrm{Ce} / \mathrm{H}]$ & 1 & - & -0.02 & 0.04 & 0.04 & -0.02 & 0.06 \\
\hline & & & & & & & \\
\hline
\end{tabular}

K, 1.30 and 1.55, respectively, for HD 218356, which are systematically lower than our values, 4459, 1.67 and 1.90. We compute the $T_{\text {eff }}$ and $\log g$ for HD 218356 using two methods, and from Table 2 we can see that they are consistent. Further in HD 218356, significant differences are found in s-process abundances between ours and MER16 estimates, which could have propagated from difference in atmospheric parameters.

The Ba abundance in four common sample are found to be lower than those of MER16, but are in good agreement with da Silva et al. (2015) for HD 202109 and Merle et al. (2014) for HR 1608. We notice that the statistical uncertainties in $\mathrm{Ba}$ abundances of MER16 are 0.18, 0.25, 0.07 and 0.22, for HD 218356, HD 202109, HR 5692, HR 1608, respectively, which are larger than errors estimated from this work. For HD 202109, [X/Fe] of most elements are consistent among four data sets within uncertainties. Especially, our values are in good agreement with da Silva et al. (2015), and the mean difference is $+0.03 \pm 0.05$ dex.

\section{DISCUSSION}

Among the six giants in our program stars, there are four known mild Ba stars, HD 13611, HD 202109, HR 5692 and HD 218356, which were classified as Ba0.3, Ba1.0, Ba0.3, and Ba2.0, respectively, by $\mathrm{Lu}$ (1991). As shown in figure 4, the s-process elements ( $\mathrm{Sr}, \mathrm{Y}, \mathrm{Zr}, \mathrm{Ba}, \mathrm{La}, \mathrm{Ce}$ and $\mathrm{Nd}$ ) are overabundant in these stars, which are consistent with literature. Two other giant stars, BD-01 469 and HR 1608, show no overabundance in s-process elements. Except HD 218356 with $[\mathrm{Fe} / \mathrm{H}]$ of -0.43 , the other five giants are distributed in a very narrow range of metallicity: $-0.09 \leq[\mathrm{Fe} / \mathrm{H}] \leq 0.02$.

\subsection{Comparison with field stars}

The abundance pattern of our sample (normal and Ba stars in binary systems) from this work are compared with the literature values of single FGK field stars from previous studies (see Figure $5 \& 6$ ), to search for differences and similarities that could help to shed some light on the origin of these objects. The literature sample for comparison consists of 90 FG disk dwarfs from Chen et al. (2000), 181 FG dwarfs from Reddy et al. (2003), 309 FGK dwarfs, subgiants and giants from da Silva et al. (2015), 276 FGK dwarfs from Mishenina et al. (2013), and a large sample of F and G dwarfs from Battistini \& Bensby (2015) (594 stars for Sc and 567 stars for Co). It is evident from Figure 5 that carbon $(\mathrm{C})$, cobalt $(\mathrm{Co})$, sodium $(\mathrm{Na}), \alpha$-elements $(\mathrm{Mg}, \mathrm{Si}, \mathrm{S}$, $\mathrm{Ca}, \mathrm{Ti}$ ), iron-peak elements ( $\mathrm{Sc}, \mathrm{V}, \mathrm{Cr}, \mathrm{Mn}, \mathrm{Ni}, \mathrm{Cu}$ ), Odd$\mathrm{Z}$ light elements $(\mathrm{Al}, \mathrm{K})$ in most of our sample stars follow the trends of field stars. The s-process elements for seven $\mathrm{Ba}$ stars in our sample show different degree of enhancements.

In figure 5 we can see that normal giant stars are underabundant in $[\mathrm{C} / \mathrm{Fe}]$ and overabundant in $[\mathrm{Na} / \mathrm{Fe}]$ in the metallicity range of $-0.3<[\mathrm{Fe} / \mathrm{H}]<0.2$. A generally used explanation for this differences is that the mixing processes brings up C-poor and Na-rich material to the surface of evolved stars.

C abundance in four Ba stars (HD 202109, $\mathrm{RE} \mathrm{J} 0702+129, \mathrm{BD}+80^{\circ} 670$ and $\left.\mathrm{BD}+68^{\circ} 1027\right)$ are just like other normal stars following the abundance distribution of field sample stars. We noticed that two Ba stars (RE J0702+129 and HD 218356) in our sample show slight overabundance of $\mathrm{Na}$ (0.12 and 0.33), whereas other stars have values of $[\mathrm{Na} / \mathrm{Fe}]$ similar to the field stars of same metallicity. da Silva et al. (2015) found that some $\mathrm{Ba}$ giants in their sample show excess of $\mathrm{Na}$. It is worth mentioning that these two stars show the signatures of being contaminated by their companion and they have not reached the AGB phase to bring up the Na-rich material to the surface through dredge-up process. Shi et al. (2004) argued that large amount of $\mathrm{Na}$ could be produced by AGB stars. Considering the total uncertainties of $[\mathrm{Na} / \mathrm{Fe}]$ of the two samples, the $\mathrm{Na}$ overabundance is not very obvious. Even so, we still speculate that the two stars might have received excess $\mathrm{Na}$ from their companion (during AGB phase) through the mass-loss mechanism.

For $\mathrm{Ba}$ and normal field stars (giants and dwarfs) in our sample, the abundance trends of $\alpha$ elements $(\mathrm{Mg}, \mathrm{Si}$, $\mathrm{Ca}$ and $\mathrm{Ti}$ ) are similar to the dwarfs and giants studied by Chen et al. (2000) and da Silva et al. (2015), showing a slight increase in abundances with decreasing the metallicity. The abundance ratio of Ca in Ba dwarfs, RE J0702+129 and $\mathrm{BD}+80^{\circ} 670$, are marginally higher $(0.09$ and 0.15$)$ than those seen in normal dwarfs. We could estimate $[\mathrm{S} / \mathrm{Fe}]$ for only nine of our sample stars, and the results are consistent with Reddy et al. (2003). A large scatter was found in Potassium (K) abundance of our sample. We can see that several sample stars are located far away from the abundance pattern of the disk dwarfs. The EWs of K I line at $7699 \AA$ for $\mathrm{BD}-01^{\circ} 469, \mathrm{BD}-01^{\circ} 343, \mathrm{BD}+20^{\circ} 5125$, RE J0702+129 and $\mathrm{BD}+39^{\circ} 539$ are from 217.0 to $379.6 \mathrm{m \AA}$, which are stronger and very sensitive to damping constants. It maybe partly responsible for the large scatter.

The abundances of iron peak elements ( $\mathrm{Sc}, \mathrm{V}, \mathrm{Cr}$, $\mathrm{Ni}$ and $\mathrm{Cu}$ ) in our sample follow the trend of field stars, and show no trend in the range of metallicities of our sample. However, the abundance trend shows a slight increase in Mn abundance with increasing the metallicity. Pereira \& Porto de Mello (1997) and Pereira et al. (1998) found two Ba-enriched symbiotic stars present remarkable $\mathrm{Cu}$ depletion. Castro et al. (1999) analyzed $\mathrm{Cu}$ and Ba abun- 

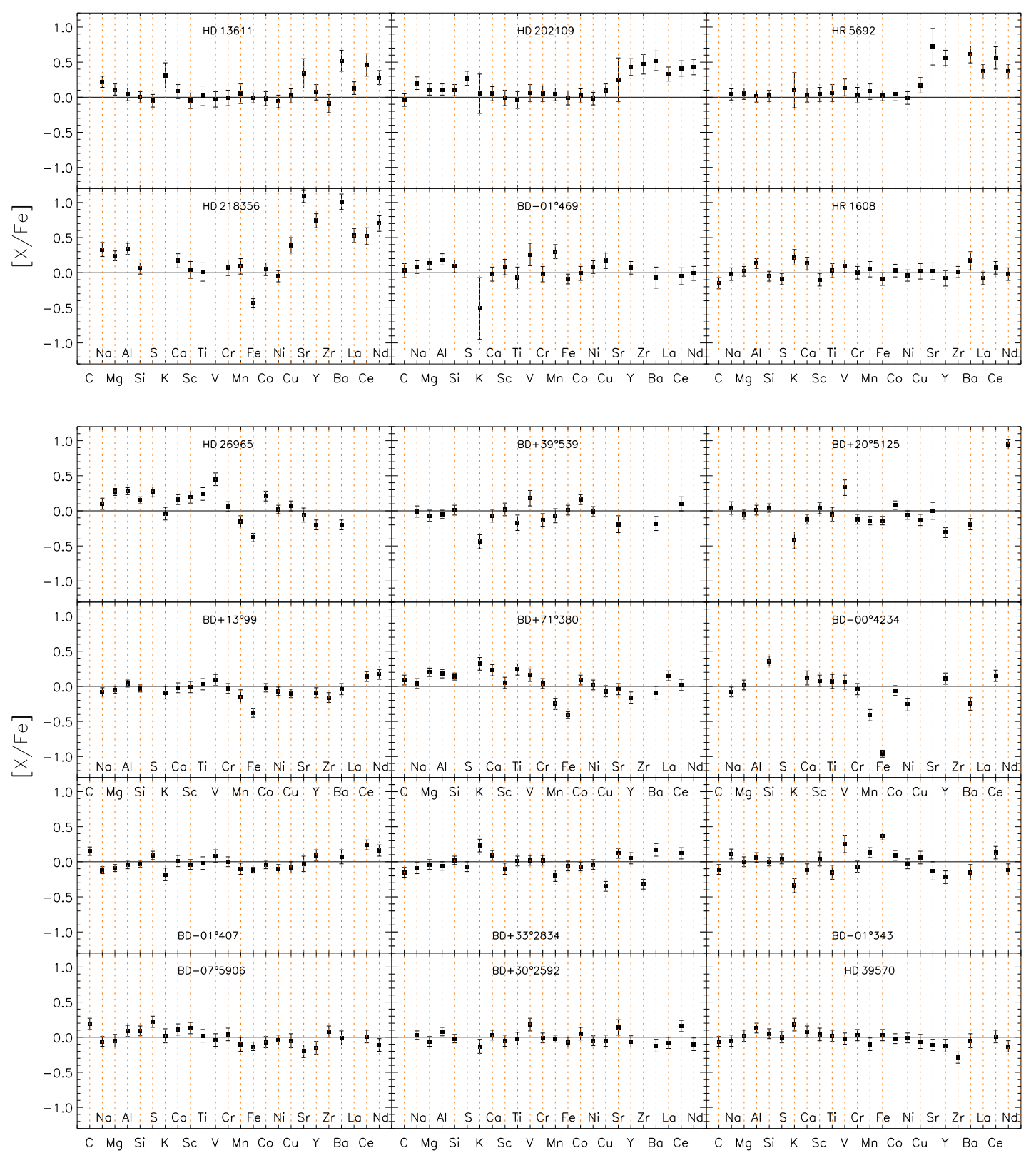

Figure 4. The abundance pattern for 18 sample stars in this work. Each abundance is given with an total error bar which has been listed in Table 8 .

dances for seven Ba dwarfs and found the deficiency of $\mathrm{Cu}$ in Ba stars compared to normal disc stars of same metallicity range. It is a possible indication that $\mathrm{Cu}$ could be a seed to the production of s-process elements. Smiljanic et al. (2007) and Allen \& Porto de Mello (2011) studied the correlation between the neutron-capture elements and iron peak elements, and verified whether they act as neutron seeds or poisons during the operation of the s-process, but did not find any supportive conclusion. In figure 5, it is clear that for three $\mathrm{Ba}$ dwarfs, the $\mathrm{Cu}, \mathrm{Ni}$, and $\mathrm{Mn}$ abundances are located at the bottom part of abundance distribution of field dwarfs, but still within the trend, which suggests the relation with heavy elements is unlikely.

We compared our $[\mathrm{Co} / \mathrm{Fe}]$ ratios with those of Reddy et al. (2003) and Battistini \& Bensby (2015), and found that the trend of our sample is consistent with those of them.

A small but distinct difference was noticed in trends between Chen et al. (2000) and Reddy et al. (2003) for [Al/Fe] against $[\mathrm{Fe} / \mathrm{H}]$. Reddy et al. (2003) showed $[\mathrm{Al} / \mathrm{Fe}]$ ratios have smooth decrease with increasing $[\mathrm{Fe} / \mathrm{H}]$ in the whole range of metallicities for their sample, but the sample of 


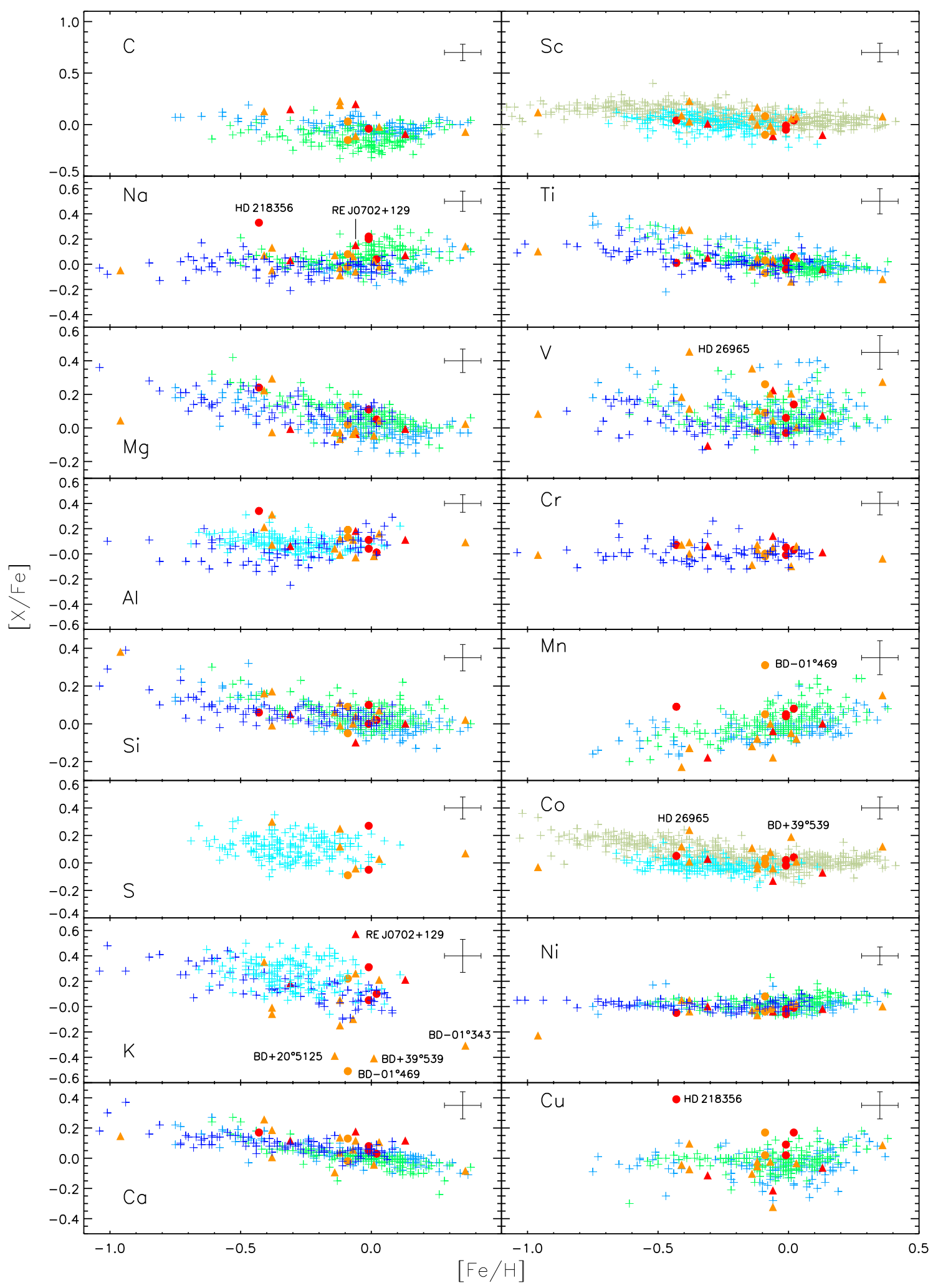

Figure 5. Abundance ratio $[\mathrm{X} / \mathrm{Fe}]$ versus $[\mathrm{Fe} / \mathrm{H}]$. Red filled circles: $\mathrm{Ba}$ giants in this work; red filled triangles: Ba dwarfs in Paper I; orange filled circles and triangles: normal giant and dwarf stars in this work; blue and green crosses: dwarfs and giants given by da Silva et al. (2015); purple crosses: $90 \mathrm{~F}$ and G disk dwarfs given by Chen et al. (2000); fluorescent blue crosses: $181 \mathrm{~F}$ and G dwarfs given by Reddy et al. (2003); Army-green crosses: F and G dwarfs in the solar neighborhood provided by Battistini \& Bensby (2015). An error bar with the average value of total errors of $[\mathrm{X} / \mathrm{Fe}]$ is shown in the top right-hand corner of each panel. 
Chen et al. (2000) showed a rather steep upturn of $[\mathrm{Al} / \mathrm{Fe}]$ beginning at $[\mathrm{Fe} / \mathrm{H}] \simeq-0.4$. Our results are in good agreement with Reddy et al. (2003) and there is no obvious difference between the giants and dwarfs in our sample.

In figure 6, we can see that seven Ba stars in our sample show overabundance of heavy elements when they are compared with the sample of similar metallicity studied by Mishenina et al. (2013). For the seven Ba stars, the abundances of s-process elements and their [s/Fe] (mean value of $\mathrm{Sr}, \mathrm{Y}, \mathrm{Zr}, \mathrm{Ba}, \mathrm{La}, \mathrm{Ce}$ and Nd) shows anti-correlation with $[\mathrm{Fe} / \mathrm{H}]$.

\subsection{Ba stars and the masses of WD companions}

The minimum CO core mass at the base of the AGB was predicted to be $0.51 M_{\odot}$ (for a star with initial mass $0.9 M_{\odot}$ ) by Eq.(66) of Hurley et al. (2000). Otherwise, the s-process synthesis would not occur in a star because the progenitor of the WD could not reach to the TP-AGB phase. MER16 analyzed 11 binary systems having WD companions of various masses and showed that the trend of s-process enrichment of their sample supported the prediction except two marginal cases, namely DR Dra $\left(0.55 M_{\odot}\right)$ and 14 Aur C (0.53 - 0.69 $\left.M_{\odot}\right)$. For 14 Aur C, MER16 indicated that the absence of s-process enrichments could be this star might have experienced the common-envelope process which does not lead to substantial accretion. For star DR Dra, the reason is still puzzling.

There are 17 binary systems whose WD masses are known in our 21 samples (including three Ba dwarfs from $\mathrm{Pa}-$ per I). Except four common stars (HD 218356, HD 202109, HR 5692 and HR 1608) we include other seven sample from MER16 to our sample, and present the average sprocess abundances against the masses of WD companions in figure 7. It is clear that four stars whose WD companion masses are less than $0.51 M_{\odot}$ do not indeed show presence of s-process enrichment, supporting the prediction of Hurley et al. (2000) and previous observations by Merle et al. (2016). Interestingly, except star DR Dra and 14 Aur C, there are still nine stars whose WD masses qualify for them being polluted by s-process elements, but they are showing absence of s-process enrichment. The largest separation between the seven $\mathrm{Ba}$ stars and their WD companions in our sample is about $3028 \mathrm{AU}$ (for $\mathrm{BD}+68^{\circ} 1027$ ), and seven samples whose WD companion masses are larger than $0.51 M_{\odot}$ and orbital separations are less than 3028 $\mathrm{AU}$ are showing the absence of s-process enrichment. Consequently, above analysis indicates a large enough mass of WD with a small enough orbital separation in binaries is not a sufficient condition to form a Ba star. On the positive side, $0.51 M_{\odot}$ is probably the threshold to indicate whether the progenitor of a WD has reach the TP-AGB phase.

\subsection{Level of s-process enrichment and the orbital separation}

The orbital separation of the binary systems might govern the efficiency of mass transfer from s-process enriched AGB stars, and it has been widely assumed to be one of the important reasons for different levels of s-process enrichment in Ba stars (Han et al. 1995; Pols et al. 2003). In this work, we use the estimated semimajor axis (see Table 1) to stand for the orbital separations. RE J0702+129 and HD 218356 are unresolved binaries, so we use zero value to represent their orbital separations. We showed [s/Fe] against orbital separations in figure 8. Our 21 program stars together with two stars from MER16 (14 Aur C and IP Eri), which had been catalogued in Holberg et al. (2013) and provided the semimajor axis, have been presented. We noticed that, except seven Ba stars, there is no apparent overabundances of s-process elements in other 16 stars. For the seven $\mathrm{Ba}$ stars, we found no obvious correlation between their $[\mathrm{s} / \mathrm{Fe}]$ ratios and orbital separations. We can conclude that the binary orbital separation is not the only reason that cause the difference between the mild and the strong Ba stars.

\subsection{Level of s-process enrichment and the metallicity}

Another possible reason that cause the difference between the mild and strong Ba stars is the efficiency of the neutroncapture nucleosynthesis in AGB stars, which is controlled by the metallicity (Jorissen et al. 1998; Käppeler et al. 2011). The $[\mathrm{hs} / \mathrm{ls}]$ ratio has been widely used to measure the sprocess efficiency since Luck \& Bond (1991). [hs] and [ls] stands for the mean abundance of 'heavy' and 'light' sprocess elements, respectively.

In figure 9 , we show $[\mathrm{hs} / \mathrm{ls}]$ ratio and $[\mathrm{s} / \mathrm{Fe}]$ ratio of our sample along with Ba stars analyzed in de Castro et al. (2016) and Pereira et al. (2011). Different set of elements were used to calculate [hs] and [ls] in literature. Here, to maintain consistency among the three data sets, we adopted $\mathrm{Zr}$ and $\mathrm{Y}$ to calculate [ls] and $\mathrm{La}, \mathrm{Ce}$ and $\mathrm{Nd}$ to calculate [hs]. 's' is the mean value of $\mathrm{Zr}, \mathrm{Y}, \mathrm{Ce}, \mathrm{Nd}$ and La. We found that there is an anti-correlation for $[\mathrm{hs} / \mathrm{ls}]$ vs. $[\mathrm{Fe} / \mathrm{H}]$ and $[\mathrm{s} / \mathrm{Fe}]$ vs. $[\mathrm{Fe} / \mathrm{H}]$, which is consistent with literature (Smiljanic et al. 2007; Pereira et al. 2011). It has been suggested in literature that the different level of s-process elemental overabundance is controlled by the metallicity. However, we found no significant difference in metallicity between strong $\mathrm{Ba}$ and mild Ba stars when we put our sample together with the data provided by de Castro et al. (2016) and Pereira et al. (2011). In order to inspect the relation between $[\mathrm{hs} / \mathrm{ls}]$ ratio and level of n-capture efficiency, in all data sets, the stars with $[\mathrm{s} / \mathrm{Fe}]$ larger than 0.8 are plotted in green color and represent 'strong Ba stars'. The others which are plotted orange color represent 'mild Ba stars'. We found no distinct separation between 'strong Ba stars' and 'mild Ba stars' in the range of $-1.0<[\mathrm{Fe} / \mathrm{H}]<0.3$. The overlap will be even more obvious if we set the threshold to be 0.6 for 'strong Ba stars'. Even so, we can see that there is a general trend for the 'mild Ba stars' to fall below the 'strong Ba stars' of the same metallicity.

\subsection{The ratio [hs/ls] and the masses of WD companions}

Karakas \& Lugaro (2016) (hereafter KL2016) predicted that low-mass AGB stars produce a higher proportion of [hs] with respect to [ls] elements at solar metallicity. Delgado Mena et al. (2017) analyzed chemical abundances of 1111 FGK dwarf stars and found that thick disc stars have 


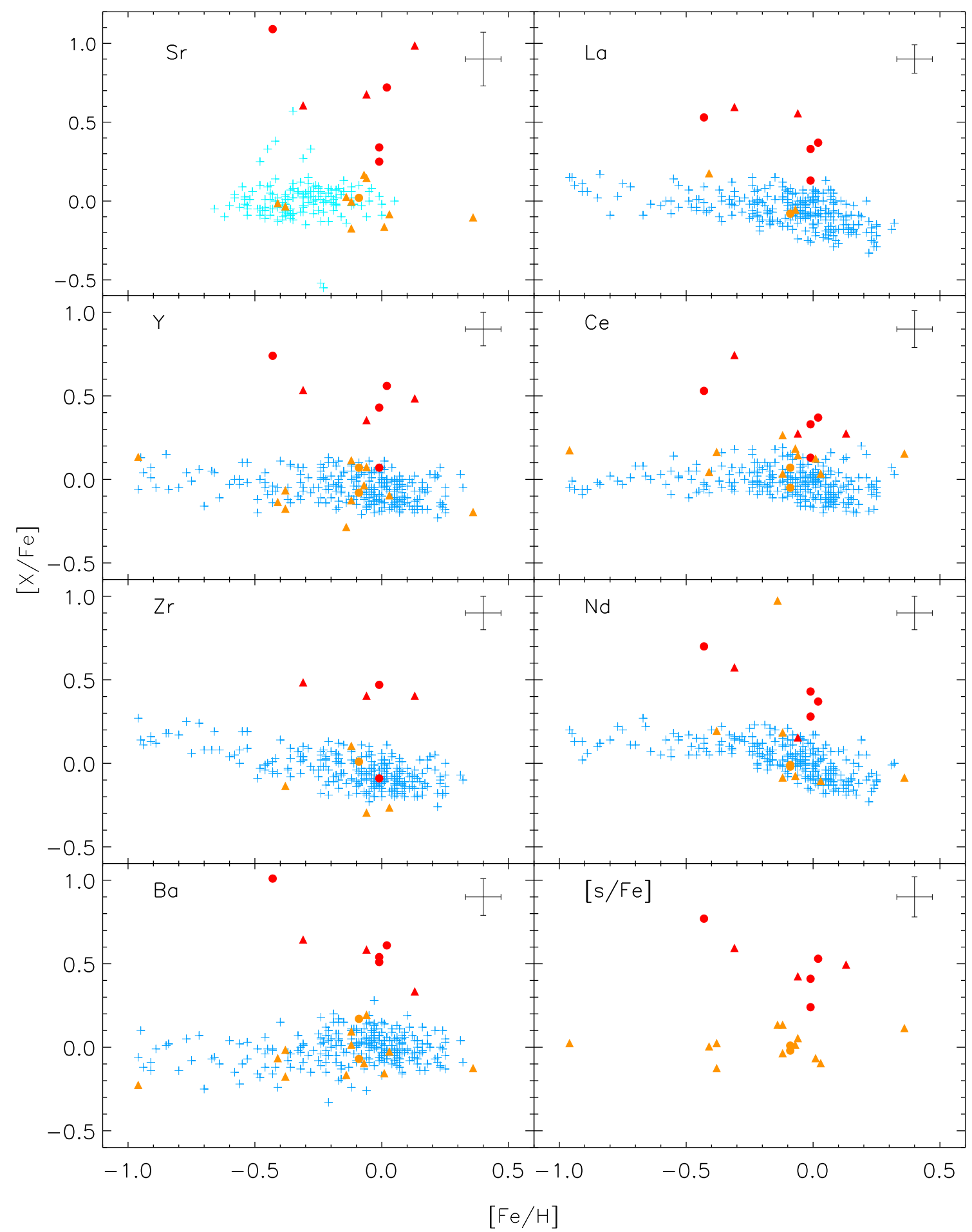

Figure 6. Abundance trends of $[\mathrm{X} / \mathrm{Fe}]$ against $[\mathrm{Fe} / \mathrm{H}]$ for seven heavy elements ( $\mathrm{Sr}, \mathrm{Y}, \mathrm{Zr}, \mathrm{Ba}, \mathrm{La}$, Ce and $\mathrm{Nd}$ ) and the mean values of them. Red filled circles and triangles: Ba giants in this work and Ba dwarfs in Paper I; orange filled circles and triangles: normal giant and dwarf stars in this work; blue crosses: 276 FGK dwarfs from Mishenina et al. (2013); fluorescent blue crosses: $181 \mathrm{~F}$ and G dwarfs from Reddy et al. (2003). An error bar with the average value of total errors of $[\mathrm{X} / \mathrm{Fe}]$ is shown in the top right-hand corner of each panel. 


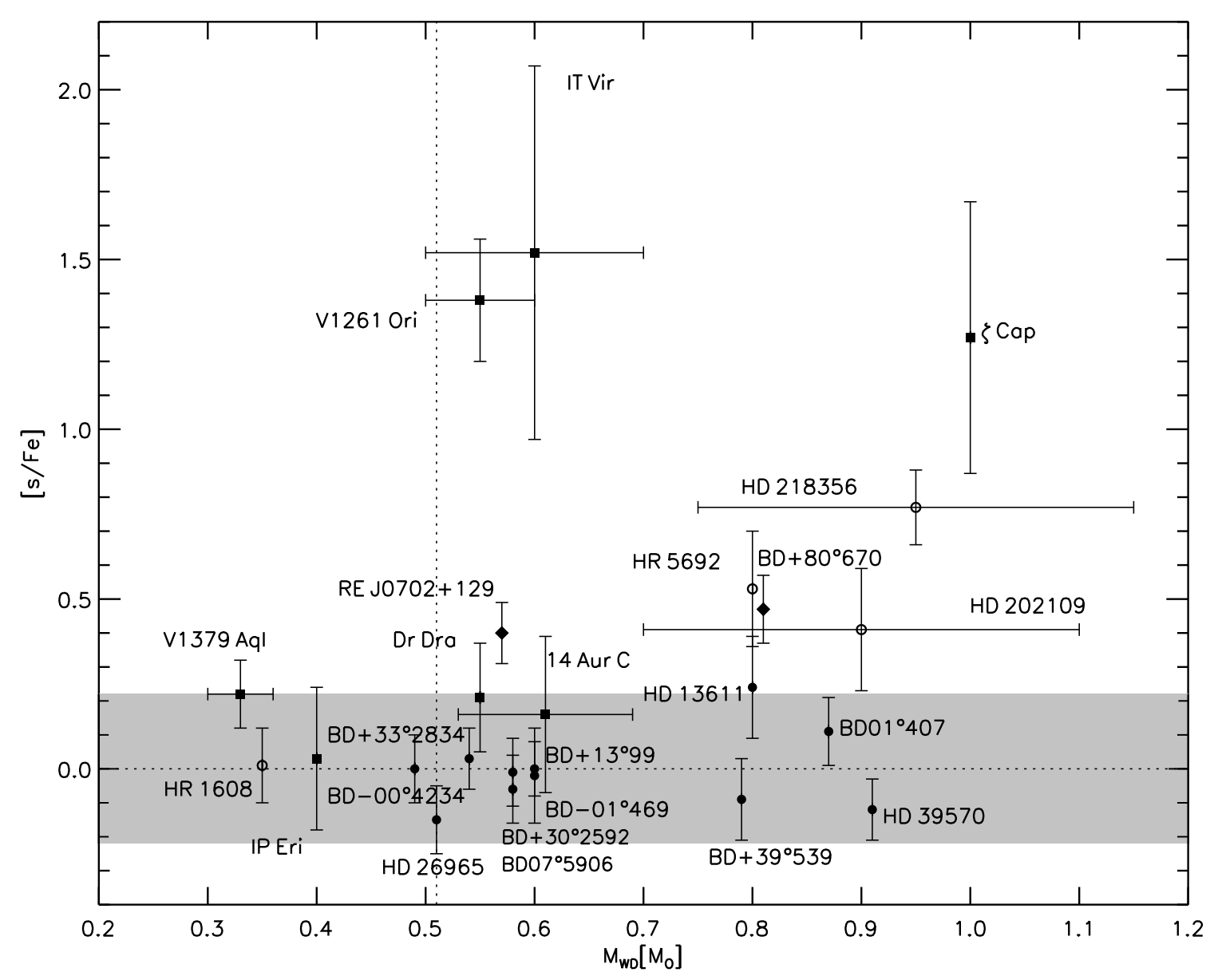

Figure 7. Average s-process abundances relative to Fe as a function of the WD masses. Total error bars are given here. Filled circles: sample stars in this work; filled rhombus: Ba dwarfs from Paper I; filled rectangle: stars from MER16; open circles: common stars in both studies. To guide the eye, the gray zone shows the \pm 0.22 dex zone without significant enhancement in s-process abundances.

less Ba with respect to light-s (lower [hs/ls]) elements compare to thin disc stars at a given metallicity. Based on the models of KL2016, they speculated that this phenomenon is probably due to old age of thick disc stars, and at the time of their formation less low-mass AGB stars (which evolve slower than intermediate-mass AGBs) might have contributed to the interstellar medium enrichment, from which they were formed. Ba stars accrete s-process enhancement material from their AGB companion, in due process, the accretion efficiency and pollution factor play a significant role (see paper I for details). After the mass transfer, the abundance pattern of AGB stars will not change but the absolute value of ratio $[\mathrm{hs} / \mathrm{ls}]$ should be smaller. Even so, in Figure 10, we still compared [hs/ls] ratio of nine Ba stars for which WD masses are available, with those models from KL2016 $(\mathrm{z}=0.014)$, in order to find whether any relation between them. For three observed stars with low metallicities, V1261 Ori $([\mathrm{Fe} / \mathrm{H}]=-0.5)$, IT Vir $([\mathrm{Fe} / \mathrm{H}]=-0.5)$ and HD $218356([\mathrm{Fe} / \mathrm{H}]=-0.43)$, we compared them with models $(\mathrm{Z}=0.007)$. The mass of AGB stars (Figure $15 \&$ 17 from KL2016 ) have been transformed to their core mass based on the models provided by KL2016. From Figure 10, we can see that, overall, the observed values are coincident with the theoretical models. The $[\mathrm{hs} / \mathrm{ls}]$ of $\mathrm{BD}+80^{\circ} 670$ is lower, which might be caused by the contribution of metal rich AGB stars that provides higher abundances of light-s elements (See Figure $10 \& 16$ in KL2016). It is worth noting that the s-process elements abundance do not show any enrichment for the models (See Table 4 in KL2016) whose initial masses are $1.25 M_{\odot}$ (core mass is $0.60 M_{\odot}$ with $\mathrm{Z}=$ 0.014 and $0.62 M_{\odot}$ with $\left.\mathrm{Z}=0.007\right)$. However, two observed stars, RE J0702+129 and V1261 Ori, whose WD companion masses are $0.57 M_{\odot}$ and $0.55 M_{\odot}$, respectively, show significant s-process elements enrichment. So, for the minimum $\mathrm{CO}$ core mass at the base of the AGB stars, our result are not matching with theoretical models of KL2016, but consistent with the prediction of Hurley et al. (2000).

\section{CONCLUSIONS}

Based on high resolution and high $\mathrm{S} / \mathrm{N}$ spectra, we carried out a detailed abundance analysis for 18 primary stars of SLSs, in which four known Ba giants, two normal giants, and 12 dwarfs. We determined atmospheric parameters, masses and abundances for 24 elements: C, Na, Mg, Al, Si, S, K, 


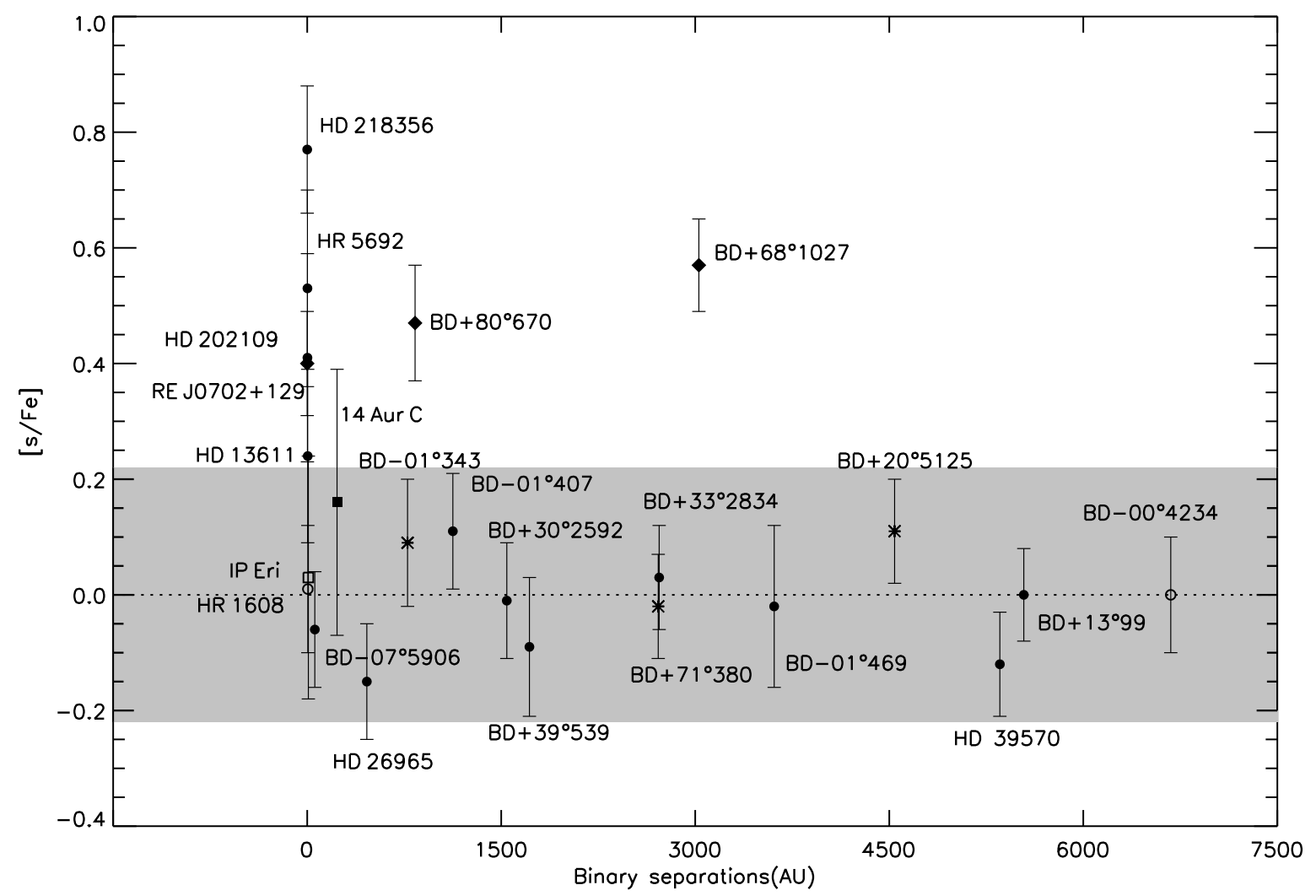

Figure 8. Abundance trends of $[\mathrm{s} / \mathrm{Fe}]$ against orbital separation (AU). Total error bars are given here. Black filled circle: sample stars with WD mass equal or larger than $0.51 M_{\odot}$ in this work; open circles: sample stars with WD mass less than $0.51 M_{\odot}$ in this work; stars: sample stars with unknown WD mass in this work; filled rhombus: Ba dwarfs from Paper I; filled rectangles: sample stars with WD mass larger than $0.51 M_{\odot}$ in MER16's work; open rectangles: sample stars with WD mass less than $0.51 M_{\odot}$ in MER16's work.

$\mathrm{Ca}, \mathrm{Sc}, \mathrm{Ti}, \mathrm{V}, \mathrm{Cr}, \mathrm{Mn}, \mathrm{Fe}, \mathrm{Co}, \mathrm{Ni}, \mathrm{Cu}, \mathrm{Sr}, \mathrm{Y}, \mathrm{Zr}, \mathrm{Ba}, \mathrm{La}, \mathrm{Ce}$, $\mathrm{Nd}$. The abundance pattern of Al, $\alpha$ elements, iron-peak elements and odd- $\mathrm{Z}$ elements of $\mathrm{Ba}$ and normal stars in binaries are similar to those of the field stars with same metallicity. We did not find any difference in the behavior of $[\mathrm{X} / \mathrm{Fe}]$ between Ba giant and dwarf stars analyzed in this study. Some stars in our sample show enhancement in sodium, compare to the field stars, and the overabundance is probably polluted by WD companions when they are in AGB phase. In previous studies, some iron peak elements have been found to have a relation with the neutron-capture elements and been predicted to act as neutron seeds or poisons during the operation of the s-process. However, we did not find any relation between the neutron-capture elements and iron peak elements.

We found correlation between s-process abundances of primary stars and masses of their WD companions in the binaries, supports the prediction made by Hurley et al. (2000), wherein s-process synthesis occur during the AGB phase when the WD mass is $0.51 M_{\odot}$ or above. So the primary star may not show s-process elemental enhancement with WD companion of mass less than $0.51 M_{\odot}$. We also found normal s-process abundances in stars with WD mass larger than $0.51 M_{\odot}$, which suggests WD mass may not be the only factor to decide the chemical peculiarities in the primary star of SLSs, complicates the present understanding of Ba stars. We did not find any relation between s-process enrichment and orbital separation of the binaries, suggests the orbital separation may not be the only reason to differentiate mild and strong Ba stars, nor a key factor to form a Ba star or not. Maybe a threshold value need to be found to reject $\mathrm{Ba}$ candidates, just like WD mass constraint, and it requires further investigation on theoretical understanding. $[\mathrm{s} / \mathrm{Fe}]$ and $[\mathrm{hs} / \mathrm{ls}]$ ratios of our sample show anti-correlation with metallicity, but we did not find any significant difference in metallicity between strong and mild Ba stars. Strong and mild Ba stars in our sample are small in number, so the threshold 0.8 is used to divide the Ba stars when including the literature sample. The correlation between the $[\mathrm{hs} / \mathrm{ls}]$ ratio and the level of s-process enhancement is not so clear, suggesting the boundary value might be a key factor. The ratio [hs/ls] as a function of WD mass is consistent with the theoretical models from KL2016. 


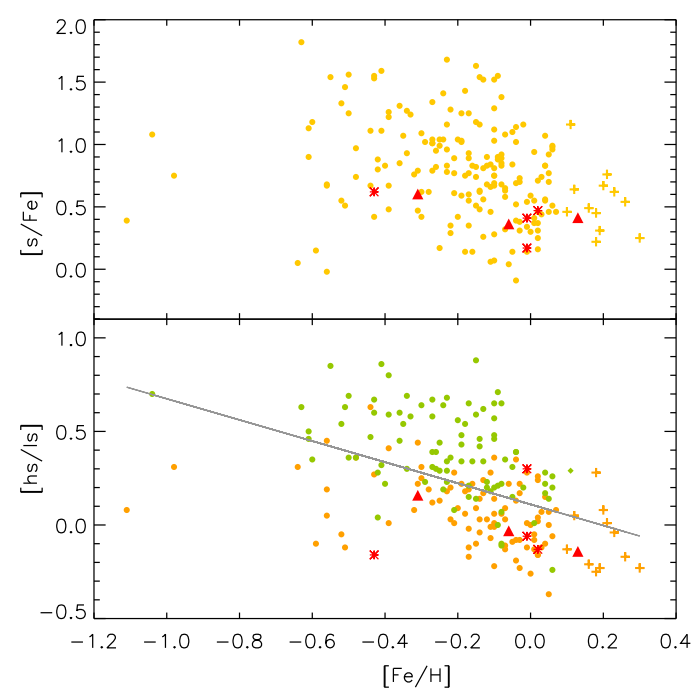

Figure 9. The ratio $[\mathrm{hs} / \mathrm{ls}]$ vs $[\mathrm{Fe} / \mathrm{H}]$ (red stars: Ba giants in this work; red triangles: Ba dwarfs in Paper I), together with the results from de Castro et al. (2016) (filled circles) and Pereira et al. (2011) (crosses). Bottom panel: green color: the sample with $[\mathrm{s} / \mathrm{Fe}]>0.8$; orange color: the sample with $[\mathrm{s} / \mathrm{Fe}]<0.8$. The solid line is a linear fit to all sample stars. For consistency with de Castro et al. (2016) and Pereira et al. (2011), for this Figure, $\mathrm{Zr}$ and $\mathrm{Y}$ are used for [ls], and La, Ce, and Nd are used for [hs]. $\mathrm{s}$ is the mean value of $\mathrm{Zr}, \mathrm{Y}, \mathrm{Ce}, \mathrm{Nd}$ and $\mathrm{La}$.

Table 6. Atmosphere parameters for the common sample stars.

\begin{tabular}{lrrrrr}
\hline \hline Star & $T_{\text {eff }}$ & $\log g$ & {$[\mathrm{Fe} / \mathrm{H}]$} & $\xi_{\mathrm{t}}$ & Ref \\
\hline HD 218356 & 4348 & 1.34 & -0.58 & 2.19 & $\mathrm{a}$ \\
& 4244 & 1.30 & -0.45 & 1.55 & $\mathrm{~b}$ \\
HD 202109 & 4459 & 1.67 & -0.43 & 1.90 & $\mathrm{~h}$ \\
& 5129 & 2.80 & 0.01 & 1.45 & $\mathrm{c}$ \\
& 4950 & 2.70 & 0.08 & 1.74 & $\mathrm{~d}$ \\
HR 5692 & 5010 & 2.68 & -0.03 & 1.43 & $\mathrm{~b}$ \\
& 5032 & 3.00 & -0.08 & 1.70 & $\mathrm{~h}$ \\
HR 1608 & 5076 & 2.86 & 0.02 & 1.35 & $\mathrm{~b}$ \\
& 5960 & 3.30 & 0.09 & 1.50 & $\mathrm{~h}$ \\
HD 13611 & 5374 & 3.60 & -0.18 & 1.03 & $\mathrm{~b}$ \\
& 5129 & 3.35 & -0.09 & 1.30 & $\mathrm{~h}$ \\
HD 26965 & 5184 & 2.45 & -0.05 & 1.63 & $\mathrm{f}$ \\
& 5153 & 4.39 & -0.31 & 1.50 & $\mathrm{~h}$ \\
& 5135 & 4.51 & -0.38 & 0.70 & $\mathrm{~g}$ \\
\end{tabular}

$a$ Bubar \& King 2010; $b$ Merle et al. 2016;

$c$ Yushchenko et al. 2004; $d$ da Silva et al. 2015;

$e$ Merle et al. 2014; $f$ Luck 2014;

$g$ Adibekyan et al. 2012; $h$ this work 


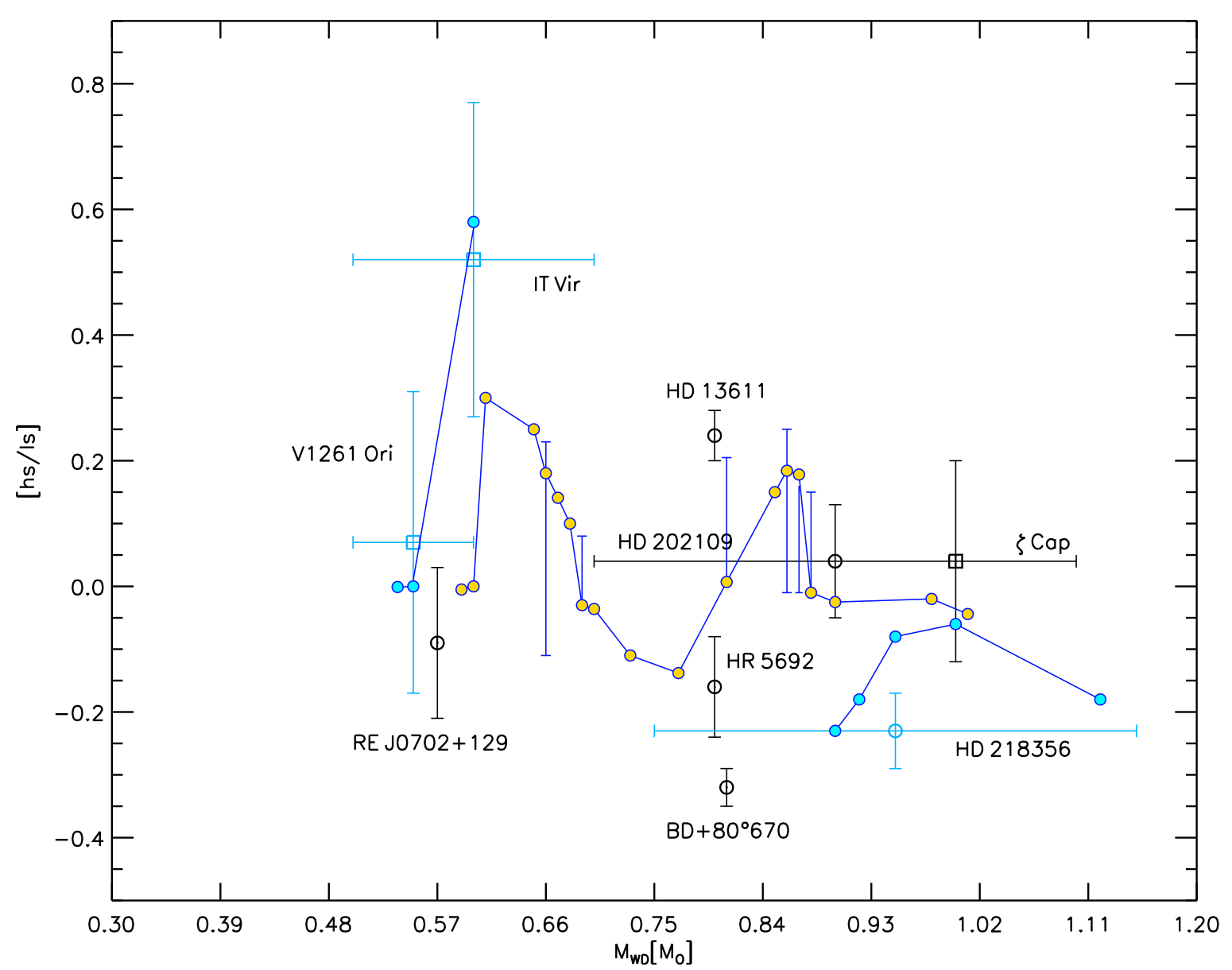

Figure 10. [hs $/ \mathrm{ls}]$ ratio as a function of WD mass. The yellow filled circles joined by blue lines represent the theoretical models from KL2016 for $\mathrm{Z}=0.014$. The filled azure circles represent the same models for $\mathrm{Z}=0.007$. For clarity, the $\mathrm{Z}=0.014$ models are only depicted close to the metal poor stars in our sample which are represented by azure open circles (this work) and rectangles (MER16). Black open rectangles and circles represent stars with solar metallicity from MER16 and this work. Total error bars are given for observed sample. 
Table 7. Abundance of the common stars. The statistical uncertainties are given here.

\begin{tabular}{|c|c|c|c|c|c|c|c|c|c|c|c|c|c|}
\hline Star & {$[\mathrm{C} / \mathrm{Fe}]$} & {$[\mathrm{Na} / \mathrm{Fe}]$} & {$[\mathrm{Mg} / \mathrm{Fe}]$} & {$[\mathrm{Al} / \mathrm{Fe}]$} & {$[\mathrm{Si} / \mathrm{Fe}]$} & {$[\mathrm{S} / \mathrm{Fe}]$} & {$[\mathrm{K} / \mathrm{Fe}]$} & {$[\mathrm{Ca} / \mathrm{Fe}]$} & {$[\mathrm{Sc} / \mathrm{Fe}]$} & {$[\mathrm{Ti} / \mathrm{Fe}]$} & {$[\mathrm{V} / \mathrm{Fe}]$} & {$[\mathrm{Cr} / \mathrm{Fe}]$} & Ref \\
\hline \multirow[t]{3}{*}{ HD 218356} & - & $0.53 \pm 0.08$ & - & $0.51 \pm 0.11$ & - & - & - & - & - & $0.07 \pm 0.24$ & - & $-0.03 \pm 0.20$ & $\mathrm{a}$ \\
\hline & $0.01 \pm 0.23$ & & - & - & - & - & - & - & - & - & - & - & b \\
\hline & - & $0.33 \pm 0.10$ & $0.24 \pm 0.10$ & $0.34 \pm 0.09$ & $0.06 \pm 0.10$ & - & - & $0.17 \pm 0.05$ & $0.04 \pm 0.09$ & $0.01 \pm 0.01$ & - & $0.07 \pm 0.02$ & $\mathrm{~h}$ \\
\hline \multirow[t]{4}{*}{ HD 202109} & - & $0.24 \pm 0.08$ & $0.22 \pm 0.22$ & $0.12 \pm 0.12$ & $-0.05 \pm 0.13$ & $0.00 \pm 0.12$ & -0.17 & $0.02 \pm 0.09$ & $0.07 \pm 0.15$ & $0.15 \pm 0.17$ & $-0.03 \pm 0.14$ & $-0.12 \pm 0.13$ & c \\
\hline & $-0.09 \pm 0.06$ & $0.18 \pm 0.09$ & $0.07 \pm 0.10$ & - & $0.02 \pm 0.09$ & - & - & $-0.01 \pm 0.12$ & - & $-0.02 \pm 0.11$ & $0 \pm 0.13$ & - & $\mathrm{d}$ \\
\hline & $-0.22 \pm 0.13$ & & - & - & - & - & - & - & - & - & - & - & $\mathrm{b}$ \\
\hline & $-0.04 \pm 0.03$ & $0.20 \pm 0.06$ & $0.11 \pm 0.06$ & $0.11 \pm 0.06$ & $0.10 \pm 0.09$ & 0.27 & 0.05 & $0.05 \pm 0.08$ & $-0.01 \pm 0.10$ & $-0.04 \pm 0.09$ & $0.06 \pm 0.06$ & $0.05 \pm 07$ & $\mathrm{~h}$ \\
\hline \multirow{2}{*}{ HR 5692} & $-0.24 \pm 0.10$ & - & & - & - & - & - & - & - & - & - & & D \\
\hline & - & $0.04 \pm 0.03$ & $0.05 \pm 0.05$ & $0.01 \pm 0.04$ & $0.02 \pm 0.07$ & - & 0.10 & $0.03 \pm 0.05$ & $0.04 \pm 0.06$ & $0.06 \pm 0.09$ & $0.14 \pm 0.06$ & $0.03 \pm$ జశ్రి: 08 & $\mathrm{~h}$ \\
\hline \multirow[t]{3}{*}{ HR 1608} & $0.08 \pm 0.16$ & $0.19 \pm 0.11$ & $0.18 \pm 0.10$ & $0.21 \pm 0.04$ & $-0.05 \pm 0.10$ & - & - & $0.19 \pm 0.04$ & - & $0.11 \pm 0.15$ & - & & e \\
\hline & $-0.12 \pm 0.18$ & & - & - & - & - & - & - & - & - & - & - & b \\
\hline & -0.15 & $-0.02 \pm 0.06$ & $0.02 \pm 0.05$ & $0.13 \pm 0.04$ & $-0.05 \pm 0.06$ & -0.09 & 0.22 & $0.13 \pm 0.07$ & $-0.10 \pm 0.06$ & $0.03 \pm 0.11$ & $0.09 \pm 0.08$ & $0.00 \pm$ 疋. .07 & $\mathrm{~h}$ \\
\hline \multirow[t]{2}{*}{ HD 13611} & -0.57 & 0.27 & -0.02 & 0.13 & 0.06 & 0.11 & - & 0.02 & -0.06 & -0.05 & -0.08 & .01 & 1 \\
\hline & - & $0.22 \pm 0.03$ & $0.11 \pm 0.06$ & $0.04 \pm 0.04$ & $0.00 \pm 0.08$ & -0.05 & 0.31 & $0.08 \pm 0.07$ & $-0.05 \pm 0.04$ & $0.02 \pm 0.08$ & $-0.03 \pm 0.01$ & $-0.01 \pm$ న్రి 09 & t \\
\hline \multirow[t]{2}{*}{ HD 26965} & - & $0.16 \pm 0.01$ & $0.25 \pm 0.10$ & $0.33 \pm 0.01$ & $0.14 \pm 0.03$ & - & - & $0.16=$ & $0.17 \pm 0.13$ & $0.37 \pm 0.11$ & $0.51 \pm 0.19$ & $0.07 \pm 0.03$ & g \\
\hline & - & $0.10 \pm 0.10$ & $0.27 \pm 0.03$ & $0.28 \pm 0.07$ & $0.15 \pm 0.06$ & 0.27 & -0.04 & $0.16 \pm 0.06$ & $0.19 \pm 0.10$ & $0.24 \pm 0.10$ & $0.43 \pm 0.07$ & $0.06 \pm 0.03$ & $\begin{array}{l}8 \\
h\end{array}$ \\
\hline
\end{tabular}

reference: the same as Table 6 
Table 7 - continued Abundance of the common stars. The statistical uncertainties are given here.

\begin{tabular}{|c|c|c|c|c|c|c|c|c|c|c|c|c|c|}
\hline Star & {$[\mathrm{Mn} / \mathrm{Fe}]$} & {$[\mathrm{Fe} / \mathrm{H}]$} & {$[\mathrm{Co} / \mathrm{Fe}]$} & {$[\mathrm{Ni} / \mathrm{Fe}]$} & {$[\mathrm{Cu} / \mathrm{Fe}]$} & {$[\mathrm{Sr} / \mathrm{Fe}]$} & {$[\mathrm{Y} / \mathrm{Fe}]$} & {$[\mathrm{Zr} / \mathrm{Fe}]$} & {$[\mathrm{Ba} / \mathrm{Fe}]$} & {$[\mathrm{La} / \mathrm{Fe}]$} & {$[\mathrm{Ce} / \mathrm{Fe}]$} & $\gtrsim[\mathrm{Nd} / \mathrm{Fe}]$ & Ref \\
\hline \multirow[t]{3}{*}{ HD 218356} & $-0.04 \pm 0.09$ & $-0.58 \pm 0.07$ & - & $-0.04 \pm 0.14$ & - & - & - & - & - & - & - & $>$ & $\mathrm{a}$ \\
\hline & & $-0.45 \pm 0.12$ & - & & - & $0.58 \pm 0.06$ & $0.46 \pm 0.22$ & $0.31 \pm 0.07$ & $1.46 \pm 0.18$ & $0.44 \pm 0.09$ & $0.16 \pm 0.09$ & - & b \\
\hline & 0.09 & $-0.43 \pm 0.11$ & $0.05 \pm 0.09$ & $-0.05 \pm 0.09$ & 0.39 & 1.09 & 0.74 & - & 1.01 & $0.53 \pm 0.01$ & 0.52 & $70 \pm 0.06$ & $\mathrm{~h}$ \\
\hline \multirow[t]{4}{*}{ HD 202109} & $-0.25 \pm 0.17$ & $0.01 \pm 0.11$ & $0.07 \pm 0.11$ & $-0.09 \pm 0.17$ & -0.01 & 0.26 & $0.48 \pm 0.16$ & $0.52 \pm 0.18$ & - & $0.51 \pm 0.20$ & $0.37 \pm 0.18$ & $42 \pm 0.17$ & c \\
\hline & $0.02 \pm 0.12$ & $0.08 \pm 0.10$ & - & $-0.02 \pm 0.10$ & $0.08 \pm 0.11$ & - & - & - & $0.40 \pm 0.09$ & - & - & - & d \\
\hline & & & - & - & - & 0.44 & $0.42 \pm 0.16$ & $0.39 \pm 0.20$ & $1.02 \pm 0.25$ & $0.40 \pm 0.08$ & $0.21 \pm 0.12$ & - & b \\
\hline & 0.04 & $-0.01 \pm 0.11$ & $0.02 \pm 0.06$ & $-0.02 \pm 0.07$ & 0.09 & 0.25 & $0.43 \pm 0.08$ & 0.47 & 0.52 & $0.33 \pm 0.07$ & $0.41 \pm 0.03$ & $843 \pm 0.10$ & $\mathrm{~h}$ \\
\hline \multirow[t]{2}{*}{ HR 5692} & - & $-0.08 \pm 0.09$ & - & - & - & 0.32 & $0.50 \pm 0.20$ & $0.64 \pm 0.19$ & $0.81 \pm 0.07$ & $0.44 \pm 0.10$ & $0.15 \pm 0.13$ & - & $\mathrm{b}$ \\
\hline & $0.08 \pm 0.04$ & $0.02 \pm 0.11$ & $0.04 \pm 0.06$ & $-0.01 \pm 0.07$ & 0.17 & 0.72 & $0.56 \pm 0.05$ & - & 0.61 & $0.37 \pm 0.10$ & $0.56 \pm 0.03$ & $0.37 \pm 0.03$ & $\mathrm{~h}$ \\
\hline \multirow[t]{3}{*}{ HR 1608} & - & $0.11 \pm 0.13$ & - & - & - & $0.09 \pm 0.04$ & $-0.12 \pm 0.11$ & $0.2 \pm 0.18$ & $0.18 \pm 0.04$ & $-0.05 \pm 0.14$ & $-0.22 \pm 0.08$ & $-0.04 \pm 0.12$ & $\mathrm{e}$ \\
\hline & - & & - & - & - & $0.10 \pm 0.01$ & $0.03 \pm 0.13$ & $0.08 \pm 0.17$ & $0.65 \pm 0.22$ & $0.20 \pm 0.06$ & $0.03 \pm 0.06$ & & b \\
\hline & $0.05 \pm 0.04$ & $-0.09 \pm 0.10$ & $0.03 \pm 0.10$ & $-0.04 \pm 0.07$ & $0.02 \pm 0.07$ & 0.02 & $-0.08 \pm 0.01$ & 0.01 & $0.17 \pm 0.10$ & -0.08 & $0.07 \pm 0.10$ & $-0.02 \pm 0.07$ & $\mathrm{~h}$ \\
\hline \multirow{2}{*}{ HD 13611} & -0.57 & 0.27 & -0.02 & 0.13 & 0.06 & 0.11 & - & 0.02 & -0.06 & -0.05 & -0.08 & -0.01 & $\mathrm{f}$ \\
\hline & $0.05 \pm 0.04$ & $-0.01 \pm 0.11$ & $-0.02 \pm 0.06$ & $-0.06 \pm 0.09$ & 0.02 & 0.34 & $0.07 \pm 0.05$ & -0.09 & 0.52 & 0.13 & $0.46 \pm 0.02$ & $0.28 \pm 0.03$ & $\mathrm{~h}$ \\
\hline \multirow[t]{2}{*}{ HD 26965} & $-0.07 \pm 0.03$ & -0.31 & $0.19 \pm 0.05$ & $0.04 \pm 0.03$ & - & - & - & - & - & - & - & - & $\mathrm{g}$ \\
\hline & $-0.15 \pm 0.04$ & $-0.38 \pm 0.11$ & $0.21 \pm 0.08$ & $0.02 \pm 0.06$ & $0.07 \pm 0.06$ & -0.06 & -0.20 & - & $-0.20 \pm 0.02$ & - & - & 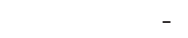 & $\mathrm{h}$ \\
\hline
\end{tabular}

reference: the same as Table 6 
Table 8. The stellar abundances of program stars. The total uncertainties are given here.

\begin{tabular}{|c|c|c|c|c|c|c|c|c|c|c|c|c|c|c|c|c|c|c|}
\hline El & HD 13611 & & HD 202109 & & HD 218356 & & HR 5692 & & $B D-01^{\circ} 469$ & & HR 1608 & & $\mathrm{BD} 00^{\circ} 4234$ & & HD 26965 & & $\mathrm{BD}-01^{\circ} 343$ & \\
\hline$[\mathrm{C} / \mathrm{Fe}]$ & & & $-0.04 \pm 0.09$ & (2) & & & & & $0.03 \pm 0.10$ & (1) & $-0.15 \pm 0.08$ & (1) & & & & & $-0.11 \pm 0.07$ & (2) \\
\hline$[\mathrm{Na} / \mathrm{Fe}]$ & $0.22 \pm 0.08$ & (2) & $0.20 \pm 0.09$ & (2) & $0.33 \pm 0.10$ & (2) & $0.04 \pm 0.08$ & (2) & $0.08 \pm 0.09$ & (2) & $-0.02 \pm 0.09$ & (3) & $-0.08 \pm 0.07$ & (2) & $0.10 \pm 0.08$ & (3) & $0.11 \pm 0.07$ & (2) \\
\hline$[\mathrm{Mg} / \mathrm{Fe}]$ & $0.11 \pm 0.08$ & (6) & $0.11 \pm 0.08$ & (6) & $0.24 \pm 0.07$ & (3) & $0.05 \pm 0.08$ & (4) & $0.13 \pm 0.08$ & (4) & $0.02 \pm 0.07$ & (5) & $0.02 \pm 0.07$ & (1) & $0.27 \pm 0.05$ & (3) & .07 & 7) \\
\hline$[\mathrm{Al} / \mathrm{Fe}]$ & $0.04 \pm 0.09$ & (5) & 88 & (5) & $0.34 \pm 0.08$ & (4) & $0.01 \pm 0.08$ & (3) & $0.19 \pm 0.08$ & (4) & $0.13 \pm 0.07$ & (6) & & & $28 \pm 0.05$ & (5) & $0.06 \pm 0.07$ & (4) \\
\hline$[\mathrm{Si} / \mathrm{Fe}]$ & $0.00 \pm 0.08$ & (20) & $0.10 \pm 0.08$ & (21) & $0.06 \pm 0.08$ & (14) & $0.02 \pm 0.08$ & (17) & $0.09 \pm 0.09$ & (17) & $-0.05 \pm 0.07$ & (23) & $0.36 \pm 0.07$ & (7) & 105 & (21) & $00 \pm 0.06$ & (18) \\
\hline$[\mathrm{S} / \mathrm{Fe}]$ & $-0.05 \pm 0.09$ & (1) & $0.27 \pm 0.10$ & (1) & & & & & & & $-0.09 \pm 0.08$ & (3) & & & $27 \pm 0.07$ & (1) & $04 \pm 0.07$ & (1) \\
\hline$[\mathrm{K} / \mathrm{Fe}]$ & $0.31 \pm 0.18$ & (1) & $0.05 \pm 0.28$ & (1) & & & $0.10 \pm 0.25$ & (1) & $-0.51 \pm 0.44$ & (1) & $0.22 \pm 0.11$ & (1) & & & $-0.04 \pm 0.09$ & (1) & $-0.34 \pm 0.10$ & (1) \\
\hline$\left[\begin{array}{l}{[\mathrm{Ca} / \mathrm{Fe}]} \\
{[\mathrm{SS} / \mathrm{Fe}]}\end{array}\right.$ & $0.08 \pm 0.10$ & (6) & $0.05 \pm 0.10$ & (7) & $0.17 \pm 0.10$ & (3) & $0.03 \pm 0.10$ & (6) & $-0.02 \pm 0.10$ & (8) & $0.13 \pm 0.09$ & (16) & $0.12 \pm 0.10$ & (3) & & (5) & $-0.11 \pm 0.08$ & 5) \\
\hline $\begin{array}{l}{[\mathrm{Sc} / \mathrm{Fe}]} \\
{[\mathrm{Ti} / \mathrm{Fe}]}\end{array}$ & $\begin{array}{r}-0.05 \pm 0.11 \\
0.02 \pm 0.14\end{array}$ & (4) & $\begin{array}{l}-0.01 \pm 0.11 \\
-0.04+0.12\end{array}$ & (8) & $\begin{array}{l}0.04 \pm 0.12 \\
0.01+0.13\end{array}$ & $\begin{array}{l}(3) \\
(5)\end{array}$ & $\begin{array}{l}0.04 \pm 0.10 \\
0.06+0.12\end{array}$ & (5) & $\begin{array}{r}0.08 \pm 0.11 \\
-0.07+0.1\end{array}$ & $(6)$ & $\begin{array}{r}-0.10 \pm 0.09 \\
0.03+0.10\end{array}$ & (8) & $0.08 \pm 0.08$ & (3) & $0.19 \pm 0.08$ & (6) & $0.04 \pm 0.10$ & $(9)$ \\
\hline $\begin{array}{l}{[\mathrm{T1} / \mathrm{Fe}]} \\
{[\mathrm{V} / \mathrm{Fe}]}\end{array}$ & $\begin{array}{r}0.02 \pm 0.14 \\
-0.03 \pm 0.11\end{array}$ & (15) & $\begin{array}{r}-0.04 \pm 0.12 \\
0.06 \pm 0.12\end{array}$ & $\begin{array}{l}(31) \\
(7)\end{array}$ & $0.01 \pm 0.13$ & (5) & $0.06 \pm 0.12$ & (14) & $\begin{array}{r}-0.07 \pm 0.15 \\
0.26+0.15\end{array}$ & (10) & $\begin{array}{l}0.03 \pm 0.10 \\
0.09+0.09\end{array}$ & (29) & $\begin{array}{l}0.07 \pm 0.10 \\
0.06+0.10\end{array}$ & $\begin{array}{l}(11) \\
(8)\end{array}$ & $0.24 \pm 0.09$ & (23) & $-0.15 \pm 0.10$ & $(27)$ \\
\hline$[\mathrm{Cr} / \mathrm{Fe}]$ & $\begin{array}{l}-0.03 \pm 0.11 \\
-0.01 \pm 0.11\end{array}$ & $\begin{array}{l}\text { (5) } \\
\text { (5) }\end{array}$ & $\begin{array}{l}0.06 \pm 0.12 \\
0.05 \pm 0.11\end{array}$ & & $0.07 \pm 0.11$ & (5) & $\begin{array}{l}0.14 \pm 0.12 \\
0.03 \pm 0.11\end{array}$ & & $\begin{array}{r}0.26 \pm 0.16 \\
-0.02 \pm 0.11\end{array}$ & & $\begin{array}{l}0.09 \pm 0.09 \\
0.00 \pm 0.09\end{array}$ & (14) & $\begin{array}{r}0.06 \pm 0.10 \\
-0.04+0.08\end{array}$ & $\begin{array}{l}(8) \\
(5)\end{array}$ & $\begin{array}{l}0.43 \pm 0.099 \\
0.06+0.07\end{array}$ & $\begin{array}{l}\text { (10) } \\
\text { (5) }\end{array}$ & $\begin{array}{r}0.25 \pm 0.12 \\
-0.07+0.08\end{array}$ & (14) \\
\hline$[\mathrm{Mn} / \mathrm{Fe}]$ & & (3) & $\begin{array}{l}0.00 \pm 0.09 \\
0.04 \pm 0.09\end{array}$ & (2) & $0.0 r \pm 0.11$ & (3) & $\begin{array}{l}0.03 \pm 0.11 \\
0.08 \pm 0.11\end{array}$ & (3) & $\begin{array}{r}-0.02 \pm 0.11 \\
0.30+0.10\end{array}$ & (1) & $\begin{array}{l}0.00 \pm 0.09 \\
0.05+0.11\end{array}$ & $\begin{array}{l}(14) \\
(3)\end{array}$ & $\begin{array}{l}-0.04 \pm 0.08 \\
-0.41+0.08\end{array}$ & (1) & $\begin{array}{r}0.06 \pm 0.07 \\
-0.15 \pm 0.08\end{array}$ & (5) & $-0.07 \pm 0.08$ & $\begin{array}{l}(14) \\
\text { (2) }\end{array}$ \\
\hline$[\mathrm{Fe} / \mathrm{I}$ & $-0.01 \pm 0.07$ & (127) & $-0.01 \pm 0.10$ & (155) & $-0.43 \pm 0.06$ & (80) & $0.02 \pm 0.07$ & (136) & $-0.09 \pm 0.07$ & (135) & $\begin{array}{r}-0.09 \pm 0.11 \\
-0.09\end{array}$ & (191) & $\begin{array}{l}-0.41 \pm 0.08 \\
-0.96 \pm 0.05\end{array}$ & (82) & $-0.38 \pm 0.06$ & (153) & $0.36 \pm 0.05$ & (117) \\
\hline$[\mathrm{Co} /$ & $-0.02 \pm 0.10$ & (7) & $0.02 \pm 0.10$ & & $0.05 \pm 0.09$ & (5) & $0.04 \pm 0.09$ & (7) & $-0.01 \pm 0.10$ & (2) & $0.03 \pm 0.09$ & (8) & $-0.06 \pm 0.07$ & (1) & $0.21 \pm 0.07$ & (10) & $0.09 \pm 0.07$ & (6) \\
\hline$[\mathrm{Ni} /$ & $-0.06 \pm 0.09$ & (33) & $-0.02 \pm 0.09$ & (35) & $-0.05 \pm 0.08$ & (16) & $-0.01 \pm 0.09$ & (38) & $0.08 \pm 0.09$ & (31) & $-0.04 \pm 0.08$ & (42) & $-0.26 \pm 0.09$ & (4) & $0.02 \pm 0.06$ & (38) & $-0.03 \pm 0.07$ & (31) \\
\hline & $0.02 \pm 0.10$ & (1) & $0.09 \pm 0.10$ & (1) & $0.39 \pm 0.11$ & (1) & $0.17 \pm 0.11$ & (1) & $0.17 \pm 0.11$ & (1) & $0.02 \pm 0.11$ & (4) & & & $0.07 \pm 0.07$ & (3) & $0.06 \pm 0.09$ & (5) \\
\hline & \pm 0.21 & (1) & 0. & (1) & $1.09 \pm 0.09$ & (1) & $0.72 \pm 0.26$ & (1) & & & $0.02 \pm$ & (1) & & & $-0.06 \pm 0.10$ & (1) & $-0.13 \pm 0.13$ & (1) \\
\hline$[\mathrm{Y} / \mathrm{H}$ & $0.07 \pm 0.11$ & (5) & 0 & (7) & $0.74 \pm 0.10$ & (3) & $0.56 \pm 0.11$ & (9) & $0.07 \pm 0.09$ & (1) & -0.08 & (2) & $0.11 \pm 0.08$ & (1) & $-0.20 \pm 0.07$ & (1) & $-0.22 \pm 0.09$ & (1) \\
\hline$[\mathrm{Zr} /$ & $-0.09 \pm$ & (1) & & (4) & & & & & & & $0 . c$ & (1) & & & & & & \\
\hline$[\mathrm{Ba} /$ & & (1) & & (1) & 1.0 & (1) & & (1) & $-0.07 \pm 0.15$ & (1) & & (3) & $-0.25 \pm 0.09$ & (1) & $-0.20 \pm 0.07$ & (3) & $-0.15 \pm 0.11$ & (2) \\
\hline$[\mathrm{La}]$ & 0.1 & (1) & 0 & (5) & & (3) & & (7) & & (1) & \pm 0.09 & (3) & & & & & & \\
\hline$[\mathrm{C}$ & $0.46 \pm 0.16$ & (2) & $0.41 \pm 0.11$ & (4) & \pm 0.12 & (2) & $0.56 \pm 0.16$ & (2) & $-0.05 \pm 0.12$ & (2) & $0.07 \pm 0.09$ & (2) & $0.15 \pm 0.08$ & (1) & & & $0.13 \pm 0.09$ & (2) \\
\hline$[\mathrm{Nd} / \mathrm{Fe}]$ & $0.28 \pm 0.10$ & (2) & $0.43 \pm 0.11$ & (5) & $0.70 \pm 0.11$ & (2) & $0.37 \pm 0.10$ & (3) & $-0.01 \pm 0.10$ & (1) & $-0.02 \pm 0.09$ & (3) & & & & & $-0.11 \pm 0.08$ & (2) \\
\hline
\end{tabular}


Table 8 - continued The differential abundances of program stars. The total uncertainties are given here.

\begin{tabular}{|c|c|c|c|c|c|c|c|c|c|c|c|c|c|c|c|c|c|c|}
\hline el & BD $-01^{\circ} 407$ & & $\mathrm{BD}+39^{\circ} 539$ & & $\mathrm{BD}-07^{\circ} 5906$ & & $\mathrm{BD}+33^{\circ} 2834$ & & $\mathrm{BD}+13^{\circ} 99$ & & $\mathrm{BD}+71^{\circ} 380$ & & $\mathrm{BD}+20^{\circ} 5125$ & & $\mathrm{BD}+30^{\circ} 2592$ & & HD 39570 & \\
\hline$[\mathrm{C} / \mathrm{Fe}]$ & $0.15 \pm 0.06$ & (1) & & & $0.19 \pm 0.08$ & (3) & $-0.15 \pm 0.07$ & (4) & & & $0.09 \pm 0.07$ & (1) & & & & & $-0.06 \pm 0.07$ & (4) \\
\hline$[\mathrm{Na} / \mathrm{Fe}]$ & $-0.12 \pm 0.05$ & (2) & $-0.01 \pm 0.08$ & (1) & $-0.06 \pm 0.07$ & (1) & $-0.09 \pm 0.08$ & (3) & $-0.08 \pm 0.06$ & (3) & $0.04 \pm 0.07$ & (3) & $0.04 \pm 0.09$ & (2) & $0.03 \pm 0.06$ & (2) & -0.0 & \\
\hline$[\mathrm{Mg} / \mathrm{Fe}]$ & $-0.09 \pm 0.05$ & (4) & $-0.07 \pm 0.08$ & (3) & $-0.05 \pm 0.09$ & (4) & $-0.04 \pm 0.07$ & (4) & $-0.05 \pm 0.05$ & (6) & 0. & (6) & & (4) & & (5) & & \\
\hline$[\mathrm{Al} / \mathrm{Fe}]$ & $-0.04 \pm 0.06$ & (4) & $-0.05 \pm 0.06$ & (6) & & (3) & & & & & & (4) & $0.01=$ & (3) & & & & \\
\hline$[\mathrm{Si} / \mathrm{Fe}]$ & $-0.03 \pm 0.05$ & (23) & $0.01 \pm 0.07$ & (11) & $0.09 \pm 0.07$ & (18) & $0.02 \pm 0.06$ & (20) & $-0.03 \pm 0$ & (21) & $0.14 \pm$ & (21) & 0.04 & & -0.02 & 0) & $05=$ & 5) \\
\hline$[\mathrm{S} / \mathrm{Fe}]$ & $0.09 \pm 0.06$ & (1) & & & $0.22 \pm 0.08$ & (3) & $-0.07 \pm 0.07$ & (2) & & & & & & & & & $0.00 \pm 0.08$ & (3) \\
\hline$[\mathrm{K} / \mathrm{Fe}]$ & $-0.18 \pm 0.09$ & (1) & $-0.44 \pm 0.10$ & (1) & $0.02 \pm 0.10$ & (1) & $0.23 \pm 0.09$ & ) & $0.09 \pm 0.09$ & $\begin{array}{lll}(1 & & \end{array}$ & $0.32 \pm 0$. & (1) & $-0.42 \pm 0.12$ & (1) & $-0.13=$ & (1) & 0.18 & ) \\
\hline $\begin{array}{l}{[\mathrm{Ca} / \mathrm{Fe}]} \\
{[\mathrm{Sc} / \mathrm{Fe}]}\end{array}$ & $0.01 \pm 0.08$ & (5) & $-0.07 \pm 0.09$ & (2) & $0.11 \pm 0.08$ & (9) & $0.09 \pm 0.07$ & (14) & -0.0 & (10) & & (11) & $-0.12=$ & (4) & & (5) & $0.08=$ & (18) \\
\hline $\begin{array}{l}{[\mathrm{Sc} / \mathrm{Fe}]} \\
{[\mathrm{Ti} / \mathrm{Fe}]}\end{array}$ & $\begin{array}{l}-0.04 \pm 0.07 \\
-0.02+0.09\end{array}$ & (4) & $\begin{array}{r}0.02 \pm 0.09 \\
-0.17+0.11\end{array}$ & (3) & $\begin{array}{l}0.13 \pm 0.08 \\
0.02+0.09\end{array}$ & (3) & $\begin{array}{r}-0.10 \pm 0.08 \\
0.01+0.07\end{array}$ & (4) & $-0.01 \pm 0.08$ & (7) & $0.05 \pm$ & (4) & $0.04 \pm$ & (7) & $-0.05=$ & & $0.04 \pm 0.09$ & (5) \\
\hline $\begin{array}{l}{[\mathrm{Ti} \mathrm{Fe}]} \\
{[\mathrm{V} / \mathrm{Fe}]}\end{array}$ & $\begin{array}{r}-0.02 \pm 0.09 \\
0.08+0.09\end{array}$ & (31) & $\begin{array}{r}-0.17 \pm 0.11 \\
0.18+0.11\end{array}$ & (9) & $\begin{array}{r}0.02 \pm 0.09 \\
-0.04+0.09\end{array}$ & (13) & $\begin{array}{l}0.01 \pm 0.07 \\
0.02+0.07\end{array}$ & (22) & $0.03 \pm 0.08$ & (27) & $0.24 \pm 0.08$ & (27) & $-0.05 \pm 0.10$ & (21) & $-0.02 \pm$ & (27) & $0.02 \pm 0.08$ & (31) \\
\hline$[\mathrm{Cr} / \mathrm{Fe}]$ & $\begin{array}{l}0.08 \pm 0.09 \\
0.00 \pm 0.07\end{array}$ & $\begin{array}{l}(8) \\
(13)\end{array}$ & $\begin{array}{r}0.18 \pm 0.11 \\
-0.13 \pm 0.09\end{array}$ & $\begin{array}{l}(4) \\
(7)\end{array}$ & $\begin{array}{r}-0.04 \pm 0.09 \\
0.04 \pm 0.09\end{array}$ & $\begin{array}{l}(6) \\
(10)\end{array}$ & $\begin{array}{l}0.02 \pm 0.07 \\
0.02+0.07\end{array}$ & $\begin{array}{l}(3) \\
(16)\end{array}$ & $\begin{array}{r}0.09 \pm 0.08 \\
-0.03+0.07\end{array}$ & $(6)$ & $0.16 \pm 0.09$ & (5) & $0.33=$ & (7) & $0.18 \pm$ & (7) & $-0.02 \pm 0.08$ & (6) \\
\hline [ Mn/ & $-0.10 \pm 0.08$ & (4) & $\begin{array}{l}-0.13 \pm 0.09 \\
-0.07 \pm 0.10\end{array}$ & (4) & $\begin{array}{r}0.04 \pm 0.09 \\
-0.10 \pm 0.10\end{array}$ & & $\begin{array}{r}0.02 \pm 0.07 \\
-0.20 \pm 0.08\end{array}$ & & $\begin{array}{l}-0.03 \pm 0.07 \\
-0.15+0.10\end{array}$ & (10) & $\begin{array}{r}0.04 \pm 0.07 \\
-0.25 \pm 0.08\end{array}$ & $\begin{array}{l}(14) \\
(4)\end{array}$ & $\begin{array}{l}-0.12 \pm 0.07 \\
-0.14 \pm 0.06\end{array}$ & (8) & $\begin{array}{l}-0.01 \pm 0.07 \\
-0.02 \pm 0.05\end{array}$ & (9) & $\begin{array}{r}0.03 \pm 0.08 \\
-0.10+0.09\end{array}$ & (15) \\
\hline$[\mathrm{Fe} / \mathrm{H}$ & $-0.12 \pm 0.04$ & (154) & $0.01 \pm 0.07$ & (54) & $-0.13 \pm 0.06$ & (131) & $-0.06 \pm 0.07$ & $(168)$ & $\begin{array}{l}-0.15 \pm 0.10 \\
-0.38 \pm 0.06\end{array}$ & (164) & $\begin{array}{l}-0.25 \pm 0.08 \\
-0.41 \pm 0.05\end{array}$ & (140) & $\begin{array}{l}-0.14 \pm 0.00 \\
-0.14 \pm 0.06\end{array}$ & (127) & $\begin{array}{l}-0.02 \pm 0.05 \\
-0.07 \pm 0.07\end{array}$ & (153) & $\begin{array}{r}-0.10 \pm 0.09 \\
0.03 \pm 0.08\end{array}$ & (205) \\
\hline$[\mathrm{Co} /$ & $-0.04 \pm 0.06$ & (8) & $0.16 \pm 0.07$ & (8) & $-0.07 \pm 0.08$ & (5) & $-0.07 \pm 0.06$ & (2) & $-0.02 \pm 0.06$ & (7) & $0.09 \pm 0.07$ & (5) & $0.08 \pm 0.06$ & (9) & $0.05 \pm 0.09$ & (5) & $-0.02 \pm 0.07$ & (5) \\
\hline$[\mathrm{Ni} / \mathrm{H}$ & $-0.10 \pm 0.06$ & (30) & $-0.01 \pm 0.07$ & (27) & $-0.04 \pm 0.07$ & (36) & $-0.04 \pm 0.07$ & (34) & $-0.07 \pm 0.06$ & (35) & $0.02 \pm 0.07$ & (34) & $-0.06 \pm 0.06$ & (37) & $-0.05 \pm 0.07$ & (42) & $-0.01 \pm 0.07$ & (40) \\
\hline$[\mathrm{Cu} /$ & $-0.08 \pm 0$ & (3) & & & -0.05 & (4) & $-0.35=$ & (3) & $-0.10 \pm 0.06$ & (2) & $-0.07 \pm 0.08$ & (2) & $-0.13 \pm$ & (2) & -0.05 & (3) & $-0.06 \pm 0.10$ & (3) \\
\hline$[\mathrm{Sr}$ & $-0.03 \pm 0.11$ & (1) & $-0.19 \pm 0.12$ & (1) & & (1) & 0.1 & (1) & & & -0.04 & (1) & 0.0 & (1) & & (1) & -0 & (1) \\
\hline$[\mathrm{Y} / \mathrm{F}$ & $0.09 \pm 0$. & (2) & & & & (3) & & (4) & -0.0 & (1) & $-0.16 \pm 0.08$ & (3) & -0.3 & (1) & 08 & (1) & 99 & ) \\
\hline$[\mathrm{Zr} / \mathrm{F}$ & & & & & & (1) & -0.3 & (1) & & (1) & & & & & & & -0 & ) \\
\hline$[\mathrm{B}$ & $0.07 \pm 0.10$ & (2) & $-0.18 \pm 0.10$ & (3) & $-0.01 \pm 0.10$ & (2) & $0.17 \pm 0.09$ & (4) & $-0.04 \pm 0.08$ & (3) & & $\begin{array}{l}\text { (3) } \\
\text { (1) }\end{array}$ & $-0.19 \pm 0.08$ & (2) & & (2) & $-0.05 \pm 0.10$ & (3) \\
\hline & & & $0.10 \pm 0.10$ & (2) & 0. & (2) & $0.12 \pm 0.08$ & (2) & $0.14 \pm 0.07$ & (1) & $0.02 \pm 0.08$ & (1) & - & & $\begin{array}{r}-0.08 \pm 0.08 \\
0.16 \pm 0.08\end{array}$ & $\begin{array}{l}(1) \\
(2)\end{array}$ & 0.09 & (2) \\
\hline$\left[\begin{array}{l}{[\mathrm{Nd} / \mathrm{Fe}]} \\
{[}\end{array}\right.$ & 0.08 & $\begin{array}{l}\text { (1) } \\
\text { (2) }\end{array}$ & $0.10 \pm 0.10$ & (2) & & (1) & $0.12 \pm 0.00$ & & 0.06 & (1) & & & 0.07 & (1) & $-0.10 \pm 0.09$ & (1) & $0.13 \pm 0.08$ & 1) \\
\hline
\end{tabular}




\section{ACKNOWLEDGEMENTS}

We are thankful to the referee for their constructive comments and suggestions that led to us improving the manuscript. We thank $\mathrm{Ji} \mathrm{Li}$ for providing help for the Observation. We also thank Yujuan Liu for valuable comments and many stimulating discussions. This study was supported by the National Natural Science Foundation of China under grant number 11390371, 11233004 and U1431106. YBK thanks the Chinese Academy of Sciences (CAS) for support through a CAS PIFI fellowship.

\section{REFERENCES}

Adibekyan V. Z., Sousa S. G., Santos N. C., Delgado Mena E., González Hernández J. I., Israelian G., Mayor M., Khachatryan G., 2012, A\&A, 545, A32

Allen D. M., Barbuy B., 2006, A\&A, 454, 917

Allen D. M., Porto de Mello G. F., 2011, A\&A, 525, A63

Alonso A., Arribas S., Martinez-Roger C., 1995, A\&A, 297, 197

Alonso A., Arribas S., Martinez-Roger C., 1996, A\&A, 313, 873

Alonso A., Arribas S., Martínez-Roger C., 1999, A\&AS, 140, 261

Alonso A., Arribas S., Martínez-Roger C., 2001, A\&A, 376, 1039

Antipova L. I., Boyarchuk A. A., Pakhomov Y. V., Panchuk V. E., 2004, Astronomy Reports, 48, 597

Battistini C., Bensby T., 2015, A\&A, 577, A9

Bidelman W. P., Keenan P. C., 1951, ApJ, 114, 473

Boffin H. M. J., Jorissen A., 1988, A\&A, 205, 155

Bubar E. J., King J. R., 2010, AJ, 140, 293

Busso M., Gallino R., Lambert D. L., Travaglio C., Smith V. V., 2001, ApJ, 557, 802

Castro S., Porto de Mello G. F., da Silva L., 1999, MNRAS, 305,693

Chen Y. Q., Nissen P. E., Zhao G., Zhang H. W., Benoni T., 2000, A\&AS, 141, 491

Cutri R. M., et al., 2003, VizieR Online Data Catalog, 2246

da Silva R., Milone A. d. C., Rocha-Pinto H. J., 2015, A\&A, 580, A24

de Castro D. B., Pereira C. B., Roig F., Jilinski E., Drake N. A., Chavero C., Sales Silva J. V., 2016, MNRAS, 459, 4299

Delgado Mena E., Tsantaki M., Adibekyan V. Z., Sousa S. G., Santos N. C., González Hernández J. I., Israelian G., 2017, preprint, (arXiv: 1705.04349)

Ferrario L., 2012, MNRAS, 426, 2500

Han Z., Eggleton P. P., Podsiadlowski P., Tout C. A., 1995, MNRAS, 277, 1443

Holberg J. B., Oswalt T. D., Sion E. M., Barstow M. A., Burleigh M. R., 2013, MNRAS, 435, 2077

Hurley J. R., Pols O. R., Tout C. A., 2000, MNRAS, 315, 543

Jorissen A., Boffin H. M. J., 1992, in Duquennoy A., Mayor M., eds, Binaries as Tracers of Star Formation. pp 110-131

Jorissen A., Van Eck S., Mayor M., Udry S., 1998, A\&A, 332, 877

Käppeler F., Gallino R., Bisterzo S., Aoki W., 2011, Reviews of Modern Physics, 83, 157

Karakas A. I., Lugaro M., 2016, ApJ, 825, 26

Kong X. M., et al., 2018, MNRAS, 474, 2129

Kovacs N., 1985, A\&A, 150, 232

Kurucz R., 1993, ATLAS9 Stellar Atmosphere Programs and 2 km/s grid. Kurucz CD-ROM No. 13. Cambridge, Mass.: Smithsonian Astrophysical Observatory, 1993., 13

Lu P. K., 1991, AJ, 101, 2229

Luck R. E., 2014, AJ, 147, 137

Luck R. E., Bond H. E., 1991, ApJS, 77, 515

McClure R. D., 1983, ApJ, 268, 264

McClure R. D., Fletcher J. M., Nemec J. M., 1980, ApJ, 238, L35

Meléndez J., et al., 2014, ApJ, 791, 14
Merle T., Jorissen A., Masseron T., Van Eck S., Siess L., Van Winckel H., 2014, A\&A, 567, A30

Merle T., Jorissen A., Van Eck S., Masseron T., Van Winckel H., 2016, A\&A, 586, A151

Mishenina T. V., Pignatari M., Korotin S. A., Soubiran C., Charbonnel C., Thielemann F.-K., Gorbaneva T. I., Basak N. Y., 2013, A\&A, 552, A128

Pereira C. B., Porto de Mello G. F., 1997, AJ, 114, 2128

Pereira C. B., Smith V. V., Cunha K., 1998, AJ, 116, 1977

Pereira C. B., Sales Silva J. V., Chavero C., Roig F., Jilinski E., 2011, A\&A, 533, A51

Pols O. R., Karakas A. I., Lattanzio J. C., Tout C. A., 2003, in Corradi R. L. M., Mikolajewska J., Mahoney T. J., eds, Astronomical Society of the Pacific Conference Series Vol. 303, Symbiotic Stars Probing Stellar Evolution. p. 290

Reddy B. E., Tomkin J., Lambert D. L., Allende Prieto C., 2003, MNRAS, 340, 304

Ryan S. G., Norris J. E., Beers T. C., 1996, ApJ, 471, 254

Shi J. R., Gehren T., Zhao G., 2004, A\&A, 423, 683

Smiljanic R., Porto de Mello G. F., da Silva L., 2007, A\&A, 468,679

Tomkin J., Lambert D. L., Edvardsson B., Gustafsson B., Nissen P. E., 1989, A\&A, 219, L15

Van der Swaelmen M., Boffin H. M. J., Jorissen A., Van Eck S., 2017, A\&A, 597, A68

Wang B., Li X.-D., Han Z.-W., 2010, MNRAS, 401, 2729

Yang G.-C., et al., 2016, Research in Astronomy and Astrophysics, 16,019

Yi S. K., Kim Y.-C., Demarque P., 2003, ApJS, 144, 259

Yushchenko A. V., Gopka V. F., Kim C., Liang Y. C., Musaev F. A., Galazutdinov G. A., 2004, A\&A, 413, 1105

Zacs L., 1994, A\&A, 283, 937

Zacs L., Musaev F. A., Bikmaev I. F., Alksnis O., 1997, A\&AS, 122

Zhao J. K., Oswalt T. D., Willson L. A., Wang Q., Zhao G., 2012, ApJ, 746, 144

Zorotovic M., Schreiber M. R., Gänsicke B. T., Nebot GómezMorán A., 2010, A\&A, 520, A86

\section{SOME EXTRA MATERIAL}

This paper has been typeset from a TEX/LATEX file prepared by the author. 